\title{
Aggregate Queries on Sparse Databases
}

\author{
Szymon Toruńczyk *
}

January 1, 2020

\begin{abstract}
We propose an algebraic framework for studying efficient algorithms for query evaluation, aggregation, enumeration, and maintenance under updates, on sparse databases. Our framework allows to treat those problems in a unified way, by considering various semirings, depending on the considered problem. As a concrete application, we propose a powerful query language extending first-order logic by aggregation in multiple semirings. We obtain an optimal algorithm for computing the answers of such queries on sparse databases. More precisely, given a database from a fixed class with bounded expansion, the algorithm computes in linear time a data structure which allows to enumerate the set of answers to the query, with constant delay between two outputs.
\end{abstract}

\section{Introduction}

The central focus of database theory is to understand the complexity of evaluating queries under various assumptions on the query and the data. There are two long lines of research in this direction. In the first line of work, the aim is to restrict the structure of the query, as measured using various width measures, such as fractional hyper-tree width or submodular width. In another line of work, pursued in this paper, structural restrictions are imposed on the data. Employing ideas from graph theory, this leads to algorithms with linear data complexity, assuming the database is sparse in some sense. Under sufficiently strong assumptions on the sparsity of the data, captured by the notion of bounded expansion, such linear-time algorithms can be obtained $[6,7]$ for all queries in first-order logic (equivalently, relational algebra).

In this paper, we consider two query languages, called weighted queries and nested weighted queries, which extend first-order logic by the ability of performing aggregation using counting, summation, minimum, and in fact, summation in arbitrary commutative semirings. An example of a weighted query is:

$$
f=\sum_{x, y, z}[E(x, y) \wedge E(y, z) \wedge E(z, x)] \cdot w(x, y) \cdot w(y, z) \cdot w(z, x) .
$$

\footnotetext{
*Institute of Informatics, University of Warsaw, Poland, szymtor@mimuw.edu.pl.
} 
Here $E$ is a binary edge relation and $w$ is a binary weight symbol. This query can be evaluated in a (directed) graph $G=(V, E)$ equipped with a binary weight function $w: V \times V \rightarrow \mathbf{S}$ taking values in an arbitrary semiring $\mathbf{S}$ (all semirings are assumed to be commutative in this paper). The operator [.] maps the boolean true to $1 \in \mathbf{S}$ and false to $0 \in \mathbf{S}$. If $\mathbf{S}$ is the semiring $(\mathbb{N},+, \cdot)$ and $w(a, b)$ represents the multiplicity of an edge $(a, b) \in E$, then the value of the query corresponds to the bag semantics of the query $\varphi(x, y, z)=E(x, y) \wedge E(y, z) \wedge E(z, x)$, i.e., the number of directed triangles in $G$, treated as a multigraph. If $\mathbf{S}$ is the semiring $(\mathbb{N} \cup\{+\infty\}$, min, +$)$ with min playing the role of addition and + of multiplication, and $w(a, b)$ represents the cost of an edge $(a, b) \in E$, then $f$ evaluates to the minimum total cost $w(a, b)+w(b, c)+w(c, a)$ of a directed triangle in $G$.

In general, a weighted query $f$ can use arbitrary first-order formulas inside the brackets [.], and the semiring operations + and $\cdot$ and aggregation (summation) $\sum$, applied to weight symbols or expressions [.]. This corresponds exactly to the positive relational algebra of Green, Karvounarakis and Tannen [9], where we allow arbitrary first-order selection predicates.

We also consider more general nested weighted queries which allow using multiple semirings within one query. An example of such a query is

$$
\max _{x}\left(\sum_{y}[E(x, y)]_{\mathbb{N}} \cdot w(y)\right) /\left(\sum_{y}[E(x, y)]_{\mathbb{N}}\right),
$$

which involves the semiring $(\mathbb{N},+, \cdot)$, used within the parenthesis, and $\mathbf{Q}_{\max }=(\mathbb{Q} \cup$ $\{-\infty\}$, max, + , with max playing the role of addition and + of multiplication, used in the outermost aggregation. Finally, $/: \mathbb{N} \times \mathbb{N} \rightarrow \mathbb{Q}$ is a connective denoting division. Given a graph $G=(V, E)$ with a unary weight function $w: V \rightarrow \mathbb{N}$, the query computes the maximum over all vertices $x$ of the average weight of the neighbors of $x$. Another example of a nested weighted query is the following query with boolean output:

$$
f(x)=\exists_{y} E(x, y) \wedge\left(w(y)>\sum_{z}[E(y, z)]_{\mathbb{N}} \cdot w(z)\right),
$$

which determines if a given node $x$ has some neighbor $y$ whose weight is larger than sum of the weights its neighbors. Here, existential quantification $\exists$ is just summation in the boolean semiring $\mathbf{B}=(\{$ true, false $\}, \vee, \wedge)$, and the inequality $>$ is treated as a connective $>: \mathbb{N} \times \mathbb{N} \rightarrow \mathbf{B}$ comparing values. Nested weighted queries extend the logic $\mathrm{FOC}(\mathbb{P})$ introduced by Kuske and Schweikardt [15].

We now describe our main results concerning the evaluation of such queries on sparse databases. Our main conceptual contribution is a framework based on circuits over semirings. A key result in this framework is an algorithm computing certain circuits representing the output of a query. As concrete applications, we obtain an efficient dynamic algorithm for evaluating weighted queries and an efficient static algorithm for evaluating weighted 
nested queries on sparse databases. To better explain and motivate our framework and its key result, we start with describing its applications.

(A) Evaluating weighted queries on sparse databases. Fix a weighted query $f$ and a database $\mathcal{D}$ equipped with several weight functions taking values in a semiring $\mathbf{S}$. The goal is to compute the value of the query $f$ on $\mathcal{D}$. We focus on the data complexity and assume the query $f$ to be fixed.

Our result gives an algorithm with linear-time data complexity for sparse input databases $\mathcal{D}$. If $f(\mathbf{x})$ is a weighted query with free variables, then the linear-time algorithm computes a data structure allowing to query the value $f(\mathbf{a})$, given a tuple $\mathbf{a} \in \mathcal{D}^{\mathbf{x}}$ in time logarithmic in $\mathcal{D}$ in general, and in constant time, if the semiring $\mathbf{S}$ is a ring or is finite. Furthermore, our algorithm is dynamic, and allows maintaining the data structure in logarithmic time whenever a weight is updated.

Our notion of sparseness is based on the Gaifman graph of the database $\mathcal{D}$, representing which elements of the database occur together in a tuple in some relation. We assume that the Gaifman graph of the input database $\mathcal{D}$ belongs to a fixed class of graphs which has bounded expansion. Classes of bounded expansion include classes of bounded maximum degree, the class of planar graphs, or every class which excludes a fixed topological minor.

(B) Evaluating nested weighted queries on sparse databases. For a broad class of nested weighted queries, denoted $\mathrm{FO}_{\mathrm{G}}[\mathbb{C}]$, we also achieve linear-time data complexity for databasese from a fixed class with bounded expansion. The query language $\mathrm{FO}_{\mathrm{G}}[\mathbb{C}]$ includes the examples of nested weighted queries mentioned above, and vastly extends a query language introduced by Grohe and Schweikardt, for which they also obtain lineartime evaluation on classes with bounded expansion, and almost linear-time evaluation for the slightly more general nowhere dense classes [12].

(C) Provenance analysis. In the two results above, we assumed the unit cost model, where semiring elements can be stored in a single memory cell, and semiring operations take constant time. Our semiring framework also allows considering complex representations of semiring elements. This has applications in provenance analysis and query enumeration, as illustrated below.

One of the aims in provenance analysis is to analyse how the tuples in the input database contribute to the answers to a query. Consider for example the query $\psi(x)=\exists_{y, z} E(x, y) \wedge$ $E(y, z) \wedge E(z, x)$. Given a graph $G=(V, E)$, assign to each edge $(a, b) \in E$ a unique identifier $e_{a b}$. Let $\mathbf{S}$ be the free commutative semiring (also called the provenance semiring), consisting of all formal sums of products of such identifiers, e.g. $e_{a b} \cdot e_{b c} \cdot e_{c a}+e_{a c} \cdot e_{c d} \cdot e_{d a}$. Define the binary weight function $w: V \times V \rightarrow \mathbf{S}$ by setting $w(a, b)=e_{a b}$. Then the weighted expression $f(x)=\sum_{y, z} w(x, y) \cdot w(y, z) \cdot w(z, x)$ evaluates at a node $a$ to the 
provenance of $a$, i.e., the element of $\mathbf{S}$ which is the sum of all products $e_{a b} \cdot e_{b c} \cdot e_{c a}$, where $a b c$ is a directed triangle in $G$.

As each element of $\mathbf{S}$ is a formal sum of possibly unbounded length, we therefore represent each such element by an enumerator which enumerates the elements of the formal sum. For a weighted query $f(\mathbf{x})$ and input database $\mathcal{D}$ whose weights are represented by as such black box enumerators, we show how to compute in linear data complexity a data structure which, given a tuple $\mathbf{a} \in \mathcal{D}^{\mathbf{x}}$, outputs an enumerator for $f_{\mathcal{D}}(\mathbf{a})$. The maximal delay between two outputs of the resulting enumerator is asymptotically equal to the maximal delay of the input enumerators. This yields applications to query enumeration, as follows.

(D) Dynamic query enumeration. Instead of analysing the tuples in the input database which contribute to the query answers, the free semiring may be used in a slightly different way, to represent the set of answers to a first-order query $\varphi(\mathbf{x})$ in a given database. For example, if $\mathbf{x}=\{x, y, z\}$, consider the weighted expression $f^{\prime}=\sum_{x, y, z}[\varphi(x, y, z)] \cdot w_{1}(x)$. $w_{2}(y) \cdot w_{3}(z)$, where for $i=1,2,3$ the weight function $w_{i}$ assigns a unique identifier $e_{a}^{i}$ to each element $a$ in the active domain. Then $f^{\prime}$ evaluates to a formal sum of products $e_{a}^{1} \cdot e_{b}^{2} \cdot e_{c}^{3}$, over all answers $(a, b, c)$ to $\varphi$. Applying the result $(\mathrm{C})$ yields a linear-time algorithm computing a constant-delay enumerator for the set of answers of a fixed first-order formula in a given input database. This reproves a result of Kazana and Segoufin [14], and strengthens it by allowing updates to $\mathcal{D}$ which preserve its Gaifman graph.

(E) Enumerating answers to nested weighted queries Our framework allows to easily combine the enumeration algorithm (D) with the result (B), yielding a linear time algorithm which computes a constant-delay enumerator for the set of answers to a booleanvalued nested weighted query $\varphi(\mathbf{x})$. As a single result, this is the crowning achievement of this paper (it does not subsume the other results in the paper, however). It gives an optimal - linear in the size of the input and output - algorithm for answering queries in an expressive language allowing aggregates in multiple semirings, on sparse databases.

(F) Circuits with permanent gates. Perhaps the most important contribution of this paper is of a more conceptual nature. It provides a unified framework, based on circuits over semirings. This framework allows to capture the complexity of evaluating weighted queries on sparse databases, in a way which is independent of the chosen semiring and the representation of its elements. By plugging in specific semirings, we easily obtain the above results, and more.

Besides usual addition and multiplication gates, our circuits have gates corresponding 
to the permanent of a rectangular matrix of semiring elements. For example,

$$
\operatorname{perm}\left[\begin{array}{ccccc}
a_{1} & a_{2} & \ldots & a_{n-1} & a_{n} \\
b_{1} & b_{2} & \ldots & b_{n-1} & b_{n} \\
c_{1} & c_{2} & \ldots & c_{n-1} & c_{n}
\end{array}\right]=\sum_{\substack{i, j, k \\
\text { distinct }}} a_{i} \cdot b_{j} \cdot c_{k},
$$

and this is naturally generalized to more rows.

Fix a weighted query $f$ without free variables. Our key result is an algorithm which compiles $f$ on a given sparse database $\mathcal{D}$ into a circuit $C_{\mathcal{D}}$. The inputs to the circuit are the weights of the tuples in the database. Once the weights are given, the circuit $C_{\mathcal{D}}$ evaluates to the value of the expression $f$ on $\mathcal{D}$ with the given weights. The circuit is computed in linear time from the input database $\mathcal{D}$, and has constant depth, fan-out, and number of rows in each permanent gate.

This result allows to reduce the problem of evaluating arbitrary weighted queries on an arbitrary class of sparse databases to the problem of computing the permanent in the considered semiring $\mathbf{S}$ of a given matrix with a fixed number of rows, which is a purely algebraic question. For example, we show that in any semiring $\mathbf{S}$, in the unit cost model, the permanent of a $k \times n$ matrix can be computed in time linear in $n$, and can be updated in constant time when $\mathbf{S}$ is a ring or is finite, and in logarithmic time in general. This yields the result (A), where the unit-cost model is assumed. To obtain the result (C) concerning the free semiring, we show that given a $k \times n$ matrix $M$ of elements of the free semiring, each represented by a constant-delay enumerator, we can compute in time linear in $n$ a constant-delay enumerator for the permanent of $M$.

Summary and organization. The organization of the paper is illustrated in Figure 1. Each of the results (A)-(F) listed above is novel and, we believe, interesting in its own rights. In our view, one of the main contributions of this paper is on a conceptual level, as it provides a unified framework for studying aggregation, enumeration, and updates based on evaluation of circuits in various semirings, and on reducing the analysis of arbitrary queries to the study of the permanent. This framework relies on the main technical contribution, the result (F). It produces a versatile and universal data structure, namely circuits, which can be used to different effects, by plugging in suitable semirings. It enables to easily derive the results (A)-(D), culminating in the result (E), which gives an efficient algorithm for enumerating the answers to queries from an expressive query language with semiring aggregates.

Due to lack of space, we can only explore a few applications of our framework in this paper. However, we believe that it offers an explanation of the tractability of various problems studied here, and in other papers [6, 7, 14, 3, 15, 12]. The proofs of the results marked $(*)$ are moved to the appendix. 


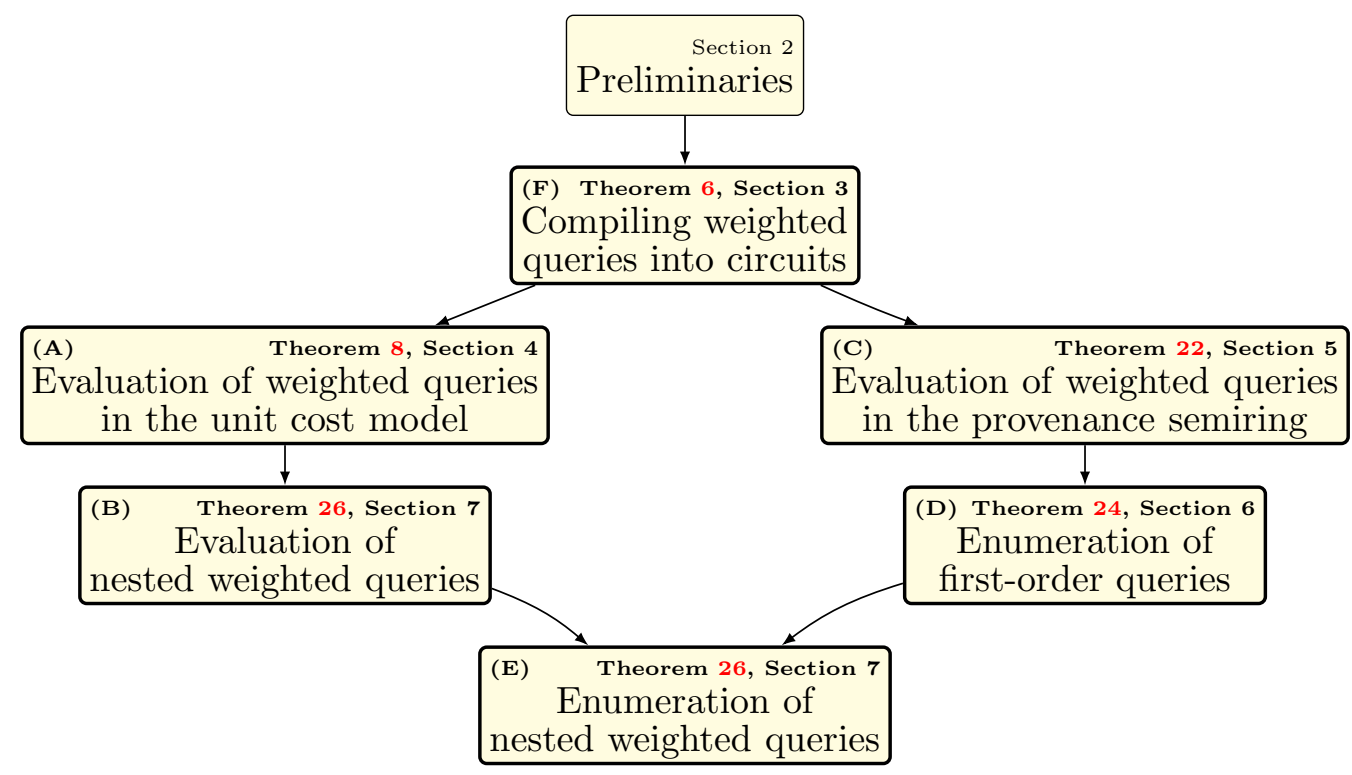

Figure 1: The results in the paper and dependencies between them. The letter in the upperleft corner corresponds to the enumeration in the introduction, and the corresponding theorem is indicated in the upper-right corner.

Background and related work. It is known from the work of Dvořák, Král', and Thomas [6, 7] that for classes with bounded expansion, model-checking first-order logic has linear data complexity. This result has been extended in multiple ways. For example, Kazana and Segoufin [14] obtained an enumeration algorithm, in which a linear-time preprocessing phase is followed by an enumeration phase which outputs all answers to the query with constant-time delay between two outputs. Both results have been extended to the slightly more general nowhere dense classes, replacing linear time by almost-linear time [11, 20]. Furthermore, Grohe and Schweikardt [12] extended the model-checking result to an extension of first-order logic by counting aggregates, denoted $\mathrm{FOC}_{1}(\mathbb{P})$. Our results (B) and (E) consider a much more powerful logic, allowing arbitrary semiring aggregates, but on the slightly less general classes with bounded expansion. In particular, we provide a partial answer to a question posed in [12, Section 9], asking if their result can be extended beyond counting aggregates. We also provide a partial answer to another question posed there, asking about enumerating answers to $\mathrm{FOC}_{1}(\mathbb{P})$ queries. Both answers are positive for classes with bounded expansion.

These results are static, i.e., any modification of the database requires recomputing 
the structure from scratch. Recently, Berkholz, Keppeler, and Schweikardt [3] obtained a dynamic version of the result of Kazana and Segoufin, maintaining the data structure in constant time each time it is modified, but under the strong restriction that the structures have bounded degree. This result was then generalized by Kuske and Schweikardt [15] to the logic with counting $\mathrm{FOC}_{1}(\mathbb{P})$. Our result $(\mathrm{D})$ is only partially dynamic, as it allows updates which preserve the Gaifman graph, but it applies to all classes of bounded expansion. However, for the special case of classes of bounded degree, the fully dynamic version follows easily, thus recovering the result [3] for first-order logic (without counting extensions).

Computing circuits for representing query outputs on sparse graphs has been employed by Amarilli, Bourhis, Mengel [1, 2]. They consider classes of bounded treewidth, which are much more restricted than classes of bounded expansion. On the other hand, they consider monadic second order (MSO) queries, which are much more powerful than first-order logic. However, they only consider the boolean semiring, and do not combine the power of MSO with other semirings.

Our circuits are very similar to, and extend, deterministic decomposable negation normal forms (d-DNNF) used in knowledge compilation [5]. They generalize them by allowing permanent gates, which, in our circuits, turn out to be both disjunctive and decomposable, in an appropriate sense. Our circuits can be alternatively viewed as factorized representations (extended by suitable permanent operators) of query answers [19].

Acknowledgements. I am very grateful to Luc Segoufin for initiating the discussion on the result (D), and am especially indebted to Micha Pilipczuk, who contributed to initial work towards that result. I would also like to thank Eryk Kopczyski, Filip Murlak, Stephan Mengel, Dan Olteanu, Sebastian Siebertz, Alexandre Vigny, and Thomas Zeume, for discussions on related topics. I am also grateful to the anonymous reviewers for useful comments.

\section{Preliminaries}

We recall the notion of classes of bounded expansion and the quantifier elimination result of Dvořák, Král', and Thomas.

\section{Graph classes of bounded expansion}

We consider undirected and simple graphs. To unclutter notation, whenever $G$ is a graph, its underlying set of nodes is also denoted $G$. We recall the notions of bounded expansion, treedepth, and low treedepth colorings.

Bounded expansion. The concept of classes of bounded expansion, proposed by Nešetřil and Ossona de Mendez in [16], captures uniform sparsity for graphs. In the original definition, a graph class $\mathscr{G}$ has bounded expansion if and only if for every $r \in \mathbb{N}$ there exists a 
constant $c_{r}$ such that the following holds: whenever we take a graph $G \in \mathscr{G}$ and construct a graph from $G$ by first removing some edges and vertices, and then contracting a collection of disjoint, connected subgraphs of radius at most $r$, then in the resulting graph, the ratio between the number of edges and the number of vertices is bounded by $c_{r}$. Intuitively, this means we require that not only the graph class $\mathscr{G}$ is sparse in the sense of admitting a linear bound on the number of edges in terms of the number of vertices, but this behavior persists even if one applies local contractions in the graph. For example, any class of bounded degree, the class of planar graphs, or any class excluding a fixed graph as a minor, has bounded expansion.

It turns out that the property of having bounded expansion is equivalent to a variety of other, seemingly unrelated conditions; see [18] for an introduction to the topic. In this work we will use the following one, which allows to decompose graphs from a class of bounded expansion into subgraphs which are essentially trees of bounded depth. This is a powerful decomposition method which allows to reduce various problems concerning classes of bounded expansion to the case of trees of bounded depth.

Low treedepth colorings. The treedepth of a graph $G$ is the minimal depth of a rooted forest $F$ with the same vertex set as $G$, such that for every edge $v w$ of $G$, either $v$ is an ancestor of $w$, or $w$ is an ancestor of $v$ in $F$. A class $\mathscr{G}$ of graphs has bounded treedepth if there is a bound $d \in \mathbb{N}$ such that every graph in $\mathscr{G}$ has treedepth at most $d$. Equivalently, $\mathscr{G}$ has bounded treedepth if there is some number $k$ such that no graph in $\mathscr{G}$ contains a path of length $k$ [18].

A class $\mathscr{G}$ has low treedepth colorings if for every $p \in \mathbb{N}$ there is a number $d \in \mathbb{N}$ and a finite set of colors $C$ such that every graph $G \in \mathscr{G}$ has a (not necessarily proper) vertex coloring $f: G \rightarrow C$ such that for any set $D \subseteq C$ of at most $p$ colors, the subgraph $G[D]$ of $G$ induced by $f^{-1}(D)$ has treedepth at most $d$.

Proposition 1 ([16]). Every class of graphs with bounded expansion has low treedepth colorings.

We remark that our notion of low treedepth colorings is more relaxed than the one from [16], but is sufficient for our needs. The converse of Proposition 1 also holds [8, Lemma 4.4], but will not be needed here.

Let us note that if $\mathscr{G}$ has bounded expansion, then for fixed $p \in \mathbb{N}$, given a graph $G \in \mathscr{G}$, a coloring $f: G \rightarrow C$ as in the definition above can be computed in linear time [16].

Example 2. We give a basic application of this powerful result, due to [17]. The proof of our key result, Theorem 6 , is an extension of this approach.

Suppose we want to answer a boolean conjunctive query $\varphi$ in a given graph $G$ from a class with bounded expansion $\mathscr{G}$. We can do this in time $\mathcal{O}_{\mathscr{C}, \varphi}(|G|)$, as follows (here, and later, the subscript in the $\mathcal{O}$ notation indicates the parameters on which the hidden constants depend on). Let $p$ be the number of variables in $\varphi$. Let $f: G \rightarrow C$ be a coloring 
as above. Then $G \models \varphi$ if, and only if, $G[D] \models \varphi$ for some $D \subseteq C$ of size $p$. Hence, it suffices to answer $\varphi$ on each of the $\mathcal{O}\left(|C|^{p}\right)=\mathcal{O}_{\mathscr{C}, \varphi}(1)$ subgraphs $G[D]$, which now have treedepth bounded by some constant $d=\mathcal{O}_{\mathscr{C}, \varphi}(1)$. This reduces the problem to the case when $\mathscr{G}$ is a class of bounded treedepth. This, in turn, reduces to labeled rooted forests of bounded depth, as follows.

If $\mathscr{G}$ is a class of graphs of treedepth at most $d$, then every graph in $G$ has maximal path length bounded by some constant $d^{\prime}$ (it is easy to show that $d^{\prime} \leqslant 2^{d}$ ). Given a graph $G$, compute in linear time a rooted spanning forest $F$ of $G$ by a depth-first search. Then $F$ has depth at most $d^{\prime}$, and for every edge $v w$ of $G$, either $v$ is an ancestor of $w$ in $F$, or vice-versa. Extend $F$ by adding unary predicates marking, for each node $v$, the depth of $v$ in $F$, and the relationship in $G$ between $v$ and each of its ancestors at depth $0,1, \ldots, d^{\prime}$. More precisely, for each $0 \leqslant i \leqslant d^{\prime}$, add unary predicates $D^{i}$ and $U^{i}$, where $D^{i}$ marks a node $v$ if it has depth $i$, and $U^{i}$ marks a node $v$ if the ancestor $w$ of $v$ in $F$ at depth $i$ is a neighbor of $v$ (if $w$ exists). It is not difficult to see that any existential formula $\varphi$ can be rewritten into an equivalent existential formula $\widehat{\varphi}$ over the signature consisting of the predicates $D^{0}, \ldots, D^{d^{\prime}}, U^{0}, \ldots, U^{d^{\prime}}$ and the parent relation, so that $G \models \varphi$ if and only if $F \models \widehat{\varphi}$. It therefore remains to solve the model-checking problem for existential formulas on labeled rooted forests of depth bounded by $d^{\prime}$ in linear time, which can be solved by a dynamic approach.

\section{Structures and logic}

We consider finite logical structures denoted $\mathcal{A}, \mathcal{B}$, etc. over a signature $\Sigma$ containing relation and/or function symbols, with the usual semantics of first-order logic, specifically, of $\Sigma$-formulas, on such structures. A structure $\mathcal{A}$ is often identified with its domain. By $|\mathcal{A}|$ we denote the size of the domain of $\mathcal{A}$. We denote finite sets of variables using symbols $\mathbf{x}, \mathbf{y}, \mathbf{z}$, etc. If $A$ is a set and $\mathbf{x}$ is a set of variables, then $A^{\mathbf{x}}$ is the set of all functions from $\mathbf{x}$ to $A$. Elements of $A^{\mathbf{x}}$ are called tuples. For such a tuple $\mathbf{a}$ and a variable $x \in \mathbf{x}$, by $\mathbf{a}[x]$ we denote the value of $\mathbf{a}$ at $x$, and for a set of variables $\mathbf{y} \subseteq \mathbf{x}$, by $\mathbf{a}[\mathbf{y}]$ we denote the restriction of $\mathbf{a}$ to $\mathbf{y}$. If $\alpha$ is a first-order formula, then we may write $\alpha(\mathbf{x})$ to indicate that the free variables of $\alpha$ are contained in $\mathbf{x}$ ( $\mathbf{x}$ may also contain additional variables). The semantics of a first-order formula $\alpha(\mathbf{x})$ in a structure $\mathcal{A}$ is the set $\alpha_{\mathcal{A}} \subseteq \mathcal{A}^{\mathbf{x}}$ of tuples which satisfy $\alpha$ in $\mathcal{A}$.

\section{Classes of structures of bounded expansion}

By the Gaifman graph of a relational structure $\mathcal{A}$ we mean the graph whose vertices are the elements of $\mathcal{A}$, and where two distinct elements $v, w$ are adjacent if and only if there is some tuple $\mathbf{a} \in R$ containing $v$ and $w$, for some relation $R$ in the signature of $\mathcal{A}$. If $\mathcal{A}$ additionally has function symbols, then its Gaifman graph is defined by considering $\mathcal{A}$ as a relational structure, by replacing each function by the relation describing its graph. 
A class of structures $\mathscr{C}$ has bounded expansion if the induced class of Gaifman graphs has bounded expansion. In this case, the number of tuples in a structure $\mathcal{A} \in \mathscr{C}$ is linear in the size of the domain, i.e., $\left|R_{\mathcal{A}}\right| \in \mathcal{O}_{\mathscr{C}}(|\mathcal{A}|)$ for each $R \in \Sigma$. Moreover, one can compute [7, Section 1.2] in time $\mathcal{O}_{\mathscr{C}}(\mathcal{A})$ a representation of $\mathcal{A}$ which allows testing membership of a given tuple in a given relation, or compute the value of a given function on a given tuple, in time $\mathcal{O}_{\mathscr{C}}(1)$. We assume this representation when working with classes of bounded expansion. Hence, for structures $\mathcal{A}$ belonging to a fixed class of bounded expansion, $|\mathcal{A}|$ can be regarded as the size of the representation of $\mathcal{A}$.

\section{Quantifier elimination on classes of bounded expansion}

The results of this paper build upon the following quantifier elimination result, which traces back to $[6,7,14,10]$. It immediately yields a model-checking algorithm for first-order logic with linear data complexity, for any class of bounded expansion.

Theorem 3. [7, Theorem 3.7] Let $\Sigma$ be a signature, $\varphi(\mathbf{x})$ a first-order $\Sigma$-formula, and $\mathscr{C}$ a class of $\Sigma$-structures of bounded expansion. There is a signature $\widehat{\Sigma}$, a quantifier-free $\widehat{\Sigma}$-formula $\widehat{\varphi}(\mathbf{x})$, a class $\widehat{\mathscr{C}}$ of $\widehat{\Sigma}$-structures of bounded expansion, and an algorithm which, given a $\Sigma$-structure $\mathcal{A} \in \mathscr{C}$, computes in time $\mathcal{O}_{\mathscr{C}, \varphi}(|\mathcal{A}|)$ a $\widehat{\Sigma}$-structure $\widehat{\mathcal{A}} \in \widehat{\mathscr{C}}$ with the same domain as $\mathcal{A}$, such that $\varphi_{\mathcal{A}}=\widehat{\varphi}_{\widehat{\mathcal{A}}}$.

Note that if $\varphi$ is a sentence then $\widehat{\varphi}$ is a quantifier-free sentence (possibly involving constants), so can be evaluated in constant time on $\widehat{\mathcal{A}}$, yielding the (boolean) value of $\varphi$ on $\mathcal{A}$. This gives the linear-time model checking algorithm.

\section{Circuits computing weighted expressions}

We define structures equipped with $\mathbf{S}$-valued weight functions, where $\mathbf{S}$ is a semiring, and define two models of computation for such structures: by means of expressions and by means of circuits with permanent gates. The main result of this section states that over classes of bounded expansion, a fixed expression can be compiled in linear time into a circuit of bounded depth and bounded fan-out.

\section{Semirings}

We only consider commutative semirings. Such a semiring $\mathbf{S}$ is a set (which we also denote S) equipped with two commutative, associative operations + and $\cdot$ with neutral elements denoted 0 and 1 respectively, where $\cdot$ distributes over + and $0 \cdot s=0$ for all $s \in \mathbf{S}$. Examples include the boolean semiring $\mathbf{B}=(\{$ true, false $\}, \vee, \wedge),(\mathbb{N},+, \cdot),(\mathbb{N} \cup\{+\infty\}, \min , \max )$, $(\mathbb{N} \cup\{+\infty\}$, min,+$)$, rings, e.g. $(\mathbb{Z},+, \cdot),(\mathbb{Q},+, \cdot)$, boolean algebras $(P(X), \cup, \cap)$, etc. 


\section{Weighted structures and expressions}

Fix a signature $\Sigma$ and a finite set of weight symbols $\mathbf{w}=\left\{w_{1}, w_{2}, \ldots\right\}$, each with a prescribed arity $r \geqslant 0$. A $\Sigma(\mathbf{w})$-structure over a semiring $\mathbf{S}$ is a $\Sigma$-structure $\mathcal{A}$ together with an interpretation of each weight symbol $w \in \mathbf{w}$ of arity $r$ as a weight function $w_{\mathcal{A}}: \mathcal{A}^{r} \rightarrow \mathbf{S}$, which only assigns nonzero weights to tuples in the structure $\mathcal{A}$. This requirement means that if $r>1$ and $w_{\mathcal{A}}(\mathbf{a}) \neq 0$ then there is some relation symbol $R \in \Sigma$ of arity $r$ such that $\mathbf{a} \in R_{\mathcal{A}}$. We write $\mathcal{A}\left(\mathbf{w}_{\mathcal{A}}\right)$ to denote the above $\Sigma(\mathbf{w})$-structure, or simply $\mathcal{A}(\mathbf{w})$ if it does not lead to confusion.

A weighted $\Sigma(\mathbf{w})$-expression, or simply $\Sigma(\mathbf{w})$-expression, is an expression such as for example:

$$
f(z)=\sum_{x}\left(w(x) \cdot \sum_{y}[(x \neq y) \wedge \neg E(x, y)] \cdot u(x, y)+w(y)\right) .
$$

Here, $[\alpha]$ denotes the Iverson bracket, which evaluates to 1 or 0 depending on whether or not the first-order formula $\alpha$ holds.

Formally, weighted expressions are defined inductively, as the smallest set of expressions containing $f_{1}+f_{2}$ and $f_{1} \cdot f_{2}$, where $f_{1}, f_{2}$ are weighted expressions, $\sum_{x} f$, where $x$ is a variable and $f$ and expression, constants $s$ for $s \in \mathbf{S}$, expressions $w\left(t^{1}, \ldots, t^{r}\right)$, where $w \in \mathbf{w}$ is a weight symbol of arity $r$ and $t^{1}, \ldots, t^{r}$ are terms built out of function symbols in $\Sigma$ and variables, and expressions of the form $[\alpha]$, where $\alpha$ is a first-order $\Sigma$-formula. We write $f(\mathbf{x})$ to underline that the free variables of $f$ are contained in $\mathbf{x}$. A closed weighted expression is a weighted expression with no free variables. A weighted expression is quantifier-free if the subexpressions $[\alpha]$ do not involve quantifiers.

Given a $\Sigma(\mathbf{w})$-structure $\mathcal{A}(\mathbf{w})$, the interpretation of an expression $f(\mathbf{x})$ in $\mathcal{A}$ is a function $f_{\mathcal{A}(\mathbf{w})}: \mathcal{A}^{\mathbf{x}} \rightarrow \mathbf{S}$, defined in the natural way. Namely, if $f(\mathbf{x})=[\alpha(\mathbf{x})]$ then $f_{\mathcal{A}(\mathbf{w})}(\mathbf{a})$ is equal to $1 \in \mathbf{S}$ if $\mathbf{a} \in \alpha_{\mathcal{A}}$ and 0 otherwise. If $f(\mathbf{x})=w\left(t^{1}, \ldots, t^{r}\right)$, then $f_{\mathcal{A}(\mathbf{w})}(\mathbf{a})=$ $w_{\mathcal{A}}\left(t_{\mathcal{A}}^{1}(\mathbf{a}), \ldots, t_{\mathcal{A}}^{r}(\mathbf{a})\right)$. If $f(\mathbf{x})=g(\mathbf{x})+h(\mathbf{x})$ then $f_{\mathcal{A}}(\mathbf{a})=g_{\mathcal{A}}(\mathbf{a})+h_{\mathcal{A}}(\mathbf{a})$, and similarly for - instead of + . Finally, if $f(\mathbf{x})=\sum_{y} g(\mathbf{x}, y)$ then $f_{\mathcal{A}(\mathbf{w})}(\mathbf{a})=\sum_{b \in \mathcal{A}} g_{\mathcal{A}(\mathbf{w})}(\mathbf{a}, b)$.

Example 4. Let $\varphi(x, y, z)$ be a first-order formula and let $\mathbf{S}=(\mathbb{Z},+, \cdot)$ be the ring of integers. Then the weighted expression $\sum_{x, y, z}[\varphi(x, y, z)]$ counts the answers to $\varphi(x, y, z)$ in a given structure $\mathcal{A}$. More generally, consider the weighted query

$$
f=\sum_{x, y, z}[\varphi(x, y, z)] \cdot p_{1}(x) \cdot p_{2}(y) \cdot p_{3}(y),
$$

where $p_{1}, p_{2}, p_{3}$ are three $\mathbb{Q}$-valued weight functions representing probability distributions on the input structure. Then $f_{\mathcal{A}}$ represents the probability that a random triple $(a, b, c)$ satisfies $\varphi(x, y, z)$, where $a, b, c$ are selected independently with distributions $p_{1}, p_{2}, p_{3}$, respectively. By a result of Kazana and Segoufin [14], this value can be computed in linear time. This is generalized in Theorem 8 , from which it is also follows that the value $f_{\mathcal{A}}$ can be updated in constant time, whenever a weight is modified. 


\section{Circuits with permanent gates}

We consider circuits whose gates perform operations from a semiring $\mathbf{S}$, and which input the weights of the tuples in the relations of a structure $\mathcal{A}$. Apart from the standard gates for addition and multiplication, the circuits may have permanent gates, implementing the permanent in $\mathbf{S}$, as defined below.

Fix a semiring $\mathbf{S}$ and let $M$ be a rectangular matrix with values in $\mathbf{S}$, i.e., $M$ is a function $M: R \times C \rightarrow \mathbf{S}$, where $R$ and $C$ are the sets of rows and columns of $M$, respectively. The permanent of $M$ is

$$
\operatorname{perm}(M)=\sum_{f} \prod_{r \in R} M[r, f(r)]
$$

where the sum ranges over all injective functions $f: R \rightarrow C$. Since $\mathbf{S}$ is commutative, the order of multiplication in (1) is irrelevant, which allows us to consider matrices with unordered sets of rows and columns. For example, if $M$ consists of three rows, $\left(a_{i}\right)_{i},\left(b_{i}\right)_{i},\left(c_{i}\right)_{i}$, then $\operatorname{perm}(M)=\sum_{i \neq j \neq k \neq i} a_{i} b_{j} c_{k}$. In our applications, the number of rows will be fixed (depending on the expression), whereas the number of columns will be unbounded (depending on the data). Note that computing the permanent of a $k \times n$ matrix amounts to evaluating the weighted expression

$$
\sum_{\substack{x_{1}, \ldots, x_{k} \\ \text { distinct }}} w_{1}\left(x_{1}\right) \cdots w_{k}\left(x_{k}\right)
$$

on the set $\{1, \ldots, n\}$ equipped with weights $w_{i}(j)=M[i, j]$ for $1 \leqslant i \leqslant k$ and $1 \leqslant j \leqslant n$.

A circuit with permanent gates is a directed acyclic graph whose nodes are called gates. If there is a directed edge from gate $g$ to gate $g^{\prime}$ then $g$ is an input to $g^{\prime}$. The size of a circuit is the number of edges plus the number of gates. The fan-in of a gate is the number of its inputs; the fan-out is defined dually. The reach-out of a gate $g$ in a circuit $C$ is the number of vertices reachable from $g$ by a directed path. The depth of $C$ is the maximal length of a directed path. The considered gates are of one of the following types:

- input gates of fan-in 0 ,

- constant gates of fan-in 0, each labeled by an element of $\mathbf{S}$,

- multiplication gates of fan-in two,

- addition gates of arbitrary fan-in, and

- permanent gates, whose inputs are indexed by pairs in $R \times C$, for some sets of rows $R$ and columns $C$. 
Note that permanent gates generalize addition and multiplication gates, respectively, by considering matrices with one row and diagonal matrices.

Given a valuation $v$ which maps the input gates to elements of a semiring $\mathbf{S}$, the value of every gate in the circuit is defined inductively in the obvious way. If a circuit $C$ has a distinguished output gate, then by $C(v)$ we denote the value at that gate induced by the valuation $v$. Note that the same circuit $C$ can be evaluated in different semirings $\mathbf{S}$, as long as they contain the constants appearing in $C$.

It will be convenient to define circuits via terms involving addition, multiplication, permanents, constants and variables. The size of a term $t$ is defined as the sum over all its subterms $t^{\prime}$ (with multiple occurrences counted once) of one plus the arity of the outermost operation in $t^{\prime}$ in case $t^{\prime}$ is not a variable or a constant. For example, the term $+(\times(y, y), \times(y, y), x, x, x)$ has size $(1+5)+(1+2)+1+1=11$. A term of size $s$ naturally defines a circuit of size $s$.

Example 5. Anticipating the main result of this section, we construct circuits evaluating the following weighted expression:

$$
f=\sum_{x, y, z}[\varphi] \cdot u(x) \cdot v(y) \cdot w(z)
$$

where $\varphi$ is the formula $x \neq y \wedge x \neq z$. For a set $A$ with weight functions $u, v, w: A \rightarrow \mathbf{S}$, we construct a circuit computing the value of $f$, whose inputs are the weights $u(a), v(a), w(a)$ of elements $a \in A$.

Observe that $\varphi$ is equivalent to the disjunction $\varphi_{1} \vee \varphi_{2}$ where $\varphi_{1} \equiv x \neq y \wedge x \neq z \wedge y \neq z$ and $\varphi_{2} \equiv x \neq y \wedge y=z$. Moreover, the disjunction $\varphi_{1} \vee \varphi_{2}$ is mutually exclusive, i.e., $\varphi_{1} \wedge \varphi_{2}$ is not satisfiable. It follows that $f$ is equivalent to the expression $f_{1}+f_{2}$, where $f_{1}$ and $f_{2}$ are obtained from $f$ by replacing $\varphi$ by $\varphi_{1}$ and $\varphi_{2}$, respectively. We construct circuits $C_{1}$ and $C_{2}$, computing the values of $f_{1}$ and $f_{2}$, respectively. The circuit $C$ computing the value of $f$ is then constructed by creating an addition gate whose inputs are the outputs of $C_{1}$ and of $C_{2}$.

Note that the value of $f_{1}$ is just the permanent of the $3 \times A$ matrix where column $a \in A$ has entries $u(a), v(a), w(a)$. This immediately yields the circuit $C_{1}$ which has one permanent gate, applied directly to the input gates, evaluating $f_{1}$ on $A$. For $f_{2}$, observe that it is equivalent to $\sum_{x, y}[x \neq y] \cdot u(x) \cdot(v(y) \cdot w(y))$. The circuit $C_{2}$ computing $f_{2}$ on $A$ is therefore a $2 \times A$ permanent gate, where column $a$ has entries $u(a)$ and $v(a) \cdot w(a)$, the latter being a multiplication gate applied to two input gates.

We now generalize the idea from Example 5 and define how circuits can evaluate weighted expressions in arbitrary structures. Fix a signature $\Sigma$ and a set of weight symbols w. We consider $\mathbf{S}$-circuits as recognizers of $\Sigma(\mathbf{w})$-structures, as follows. A $\Sigma(\mathbf{w})$-circuit over a structure $\mathcal{A}$ is an $\mathbf{S}$-circuit $C_{\mathcal{A}}$ whose inputs are pairs $(w, \mathbf{a})$, where $w \in \mathbf{w}$ is a weight symbol of arity $r$ and $\mathbf{a}$ is an $r$-tuple of elements which belongs to some relation $R_{\mathcal{A}}$ if $r>1$. 
A $\Sigma(\mathbf{w})$-structure $\mathcal{A}\left(\mathbf{w}_{\mathcal{A}}\right)$ determines a valuation of the input gates of $C_{\mathcal{A}}$ in $\mathbf{S}$, where the value assigned to the gate $(w, \mathbf{a})$ is $w_{\mathcal{A}}(\mathbf{a})$. We write $C_{\mathcal{A}}\left(\mathbf{w}_{\mathcal{A}}\right)$ to denote the output of the circuit $C_{\mathcal{A}}$ induced by this valuation. Hence, $\mathcal{A}$ is considered to be fixed, whereas the weights $\mathbf{w}_{\mathcal{A}}$ may vary, and are the inputs to the circuit.

Let $\mathscr{C}$ be a class of $\Sigma$-structures and let $\left(C_{\mathcal{A}}\right)_{\mathcal{A} \in \mathscr{C}}$ be a circuit family such that $C_{\mathcal{A}}$ is a $\Sigma(\mathbf{w})$-circuit over $\mathcal{A}$, and let $f$ be a closed $\Sigma(\mathbf{w})$-expression. We say that $\left(C_{\mathcal{A}}\right)_{\mathcal{A} \in \mathscr{C}}$ computes $f$ if for every $\Sigma$-structure $\mathcal{A}$ with $\mathcal{A} \in \mathscr{C}$, every semiring $\mathbf{S}$, and weight functions $w_{\mathcal{A}}: \mathcal{A} \rightarrow \mathbf{S}$ for $w \in \mathbf{w}$,

$$
C_{\mathcal{A}}\left(\mathbf{w}_{\mathcal{A}}\right)=f_{\mathcal{A}\left(\mathbf{w}_{\mathcal{A}}\right)} .
$$

The family $\left(C_{\mathcal{A}}\right)_{\mathcal{A} \in \mathscr{C}}$ is linear-time computable if there is an algorithm which given $\mathcal{A} \in \mathscr{C}$ outputs $C_{\mathcal{A}}$ in time $\mathcal{O}_{f, \mathscr{C}}(|\mathcal{A}|)$.

\section{Circuits for sparse structures}

We are ready to state the main result of this section. Here and later $(*)$ indicates that the proof is in the appendix.

Theorem $6(*)$. Fix a signature $\Sigma$, a set of weight symbols $\mathbf{w}$, and a closed $\Sigma(\mathbf{w})$-expression $f$. Let $\mathscr{C}$ be a class of $\Sigma$-structures of bounded expansion. There is a linear-time computable family $\left(C_{\mathcal{A}}\right)_{\mathcal{A} \in \mathscr{C}}$ of $\Sigma(\mathbf{w})$-circuits with permanent gates which computes $f$ and has bounded depth, bounded fan-out, and bounded number of rows in the permanent gates. The constants implicit in the above statements depend only on the expression $f$ and on the class $\mathscr{C}$.

Note that the same circuit $C_{\mathcal{A}}$ can be used to evaluate $f$ in an arbitrary semiring $\mathbf{S}$. This universal property captures a strong structural property of the circuits $C_{\mathcal{A}}$, which will be then exploited for enumerating answers to first-order queries without repetitions.

Theorem 6 is the cornerstone of our framework, and will allow us to reduce various problems concerning classes of bounded expansion to an algebraic analysis of the permanent over a suitably chosen semiring. This will be illustrated in the following sections.

Example 7. Let $\Sigma$ consist of a unary function $f$ and a unary predicate $R$, and let $\mathscr{C}$ be the class of forests of depth 1 , treated as $\Sigma$-structures, where in given a forest, $R$ marks all the roots and $f$ maps each non-root node to its parent and each root to itself. Consider the weighted expression

$$
f=\sum_{x, y}[\varphi] \cdot u(x) \cdot v(y)
$$

where $\varphi$ is a quantifier-free $\Sigma$-formula. Given a forest $F$, we construct a circuit $C_{F}$ evaluating $f_{F}$ on $F$, as obtained from Theorem 6 .

The formula $\varphi$ can be written as a mutually exclusive disjunction of quantifier-free types, fully specifying the relationship between $x$ and $y$ (up to equivalence in $\mathscr{C}$ ). Examples of 
such quantifier-free types are:

$$
\begin{aligned}
& \varphi_{1} \equiv R(x) \wedge R(y) \wedge x \neq y, \\
& \varphi_{2} \equiv \neg R(x) \wedge \neg R(y) \wedge x \neq y \wedge f(x)=f(y), \\
& \varphi_{3} \equiv \neg R(x) \wedge \neg R(y) \wedge f(x) \neq f(y) .
\end{aligned}
$$

Then $f$ is equivalent to the sum of expressions obtained from $f$ by substituting $\varphi$ for the quantifier-free types occurring in the disjunction. Hence, it suffices to consider the case when $\varphi$ is a quantifier-free type, as we can then add up the outputs of the circuits obtained for such expressions $f$.

For example, in the case when $\varphi=\varphi_{2}$, note that

$$
f_{F(\mathbf{w})}=\sum_{r \in R} \sum_{\substack{a, b \in f^{-1}(r) \\ a \neq b}} u(a) \cdot v(b),
$$

which readily yields a circuit: a summation gate of fan-in $|R|$, whose input corresponding to $r \in R$ is a permanent gate applied to a $2 \times f^{-1}(r)$-matrix of input gates.

When $\varphi=\varphi_{3}$, note that

$$
f_{F(\mathbf{w})}=\sum_{\substack{r, s \in R \\ r \neq s}}\left(\sum_{a \in f^{-1}(r)} u(a)\right) \cdot\left(\sum_{b \in f^{-1}(r)} v(b)\right),
$$

which yields a circuit with a $2 \times R$-permanent gate, whose inputs are summation gates.

Proof ( Sketch for Theorem 6). The proof proceeds as follows. By Theorem 3, it is enough to consider only the case when $f$ is quantifier-free, i.e., its subexpressions $[\alpha]$ do not involve quantifiers. For such expressions $f$, we prove the result in special cases of increasing generality:

Case 1: $\mathscr{C}$ is a class of vertex-labelled forests of bounded depth, where each forest is treated as a structure with unary predicates for representing the labelling, and the function mapping each non-root node to its parent. This case generalizes Example 7, and proceeds by induction on the depth.

Case 2: $\mathscr{C}$ is a class of structures of bounded treedepth, over a relational signature with binary relations only. This case reduces to the previous case, by encoding structures of bounded treedepth as labelled forests of bounded depth, similarly as in Example 2.

Case 3: $\mathscr{C}$ is a class of structures over a relational signature with binary relations only. This case reduces to the previous case, using the fact that the graphs admit low treedepth colorings, similarly as in Example 2.

Case 4: $\mathscr{C}$ is a class of structures of bounded expansion. This case reduces to the previous one, using an encoding of structures from a bounded expansion class by colored graphs. 


\section{Weighted query evaluation}

Thanks to Theorem 6, many algorithmic problems concerning the evaluation of an expression $f$ on a class of $\Sigma(\mathbf{w})$-structures boil down to analogous problems for permanents. For example, if the permanent of a $k \times n$ matrix over a semiring $\mathbf{S}$ can be computed in time $\mathcal{O}_{k}(n)$, then the same holds for the evaluation of any $\Sigma(\mathbf{w})$-expression $f$ over any $\Sigma$-structure $\mathcal{A}$ from a fixed class with bounded expansion. This turns out to be the case for any semiring $\mathbf{S}$, assuming the unit cost model. Similarly, if there is a dynamic algorithm which maintains the value of the permanent of a matrix $M$ in constant time upon singleentry modifications to $M$, then there is a dynamic algorithm which maintains in constant time the value of $f_{\mathcal{A}(\mathbf{w})}$ upon updates to the weights $\mathbf{w}$. This turns out to be the case for example if $\mathbf{S}$ is a ring or is finite.

In this section, we study how permanents can be efficiently computed and updated in various semirings. This will yield the following result, in which we consider updates to single weights.

Theorem 8. Fix a $\Sigma(\mathbf{w})$-expression $f(\mathbf{x})$, a semiring $\mathbf{S}$ whose operations take constant time, and a class $\mathscr{C}$ of $\Sigma$-structures of bounded expansion. There is an algorithm which, given a $\Sigma(\mathbf{w})$-structure $\mathcal{A}(\mathbf{w})$ with $\mathcal{A} \in \mathscr{C}$, computes in linear time a dynamic data structure allowing to query the value $f_{\mathcal{A}(\mathbf{w})}(\mathbf{a})$ at a given tuple $\mathbf{a} \in \mathcal{A}^{\mathbf{x}}$ in logarithmic time, and to maintain updates to the weights $\mathbf{w}$ in logarithmic time. If $\mathbf{S}$ is a ring or a finite semiring, then querying and updating is done in constant time.

Example 9. Consider the following weighted query ${ }^{1}$, evaluated in the ring of rationals, which computes the weights in a subsequent round of the PageRank algorithm on a directed graph $G=(V, E)$, given the weights from the previous round.

$$
f(x)=\frac{1-d}{N}+d \cdot \sum_{y}[E(y, x)] \cdot \frac{w(y)}{l(y)} .
$$

Here, $w(a)$ denotes the weight in the previous round, and $l(a)$ is the out-degree of $a, N$ is the total number of pages and $d \leqslant 1$ is a damping factor. Formally, as we don't allow division, we represent $1 / l(\cdot)$ as a weight function. Theorem 8 yields an algorithm which computes in linear time a dynamic data structure which allows to query the value $f(a)$ in constant time, and maintains updates to weights in constant time.

To prove the theorem, we analyze the permanent over various semirings. Throughout Section 4, we assume that the semiring operations of $\mathbf{S}$ take constant time.

\footnotetext{
${ }^{1}$ We thank an anonymous reviewer for suggesting this query.
} 


\section{Arbitrary semirings}

To prove Theorem 8, we consider first the case when $f$ has no free variables, as the general case reduces to this case. We show that there is an algorithm computing the value $f_{\mathcal{A}(\mathbf{w})}$ in linear time. Additionally, the value can be maintained in logarithmic time, upon updates to $\mathbf{w}$. We start by showing this for the permanent gates. In fact, we show that permanent gates can be replaced by circuits in which every gate can reach only a logarithmic number of other gates. This allows to update the output of the circuit in logarithmic time, when a single input is modified.

We remark that the evaluation of permanents of rectangular matrices in commutative semirings has been studied before, see [4] for an overview. Our algorithm below allows computing the permanent of a $k \times n$ matrix in time $2^{\mathcal{O}(k \log k)} \cdot n$. The dependency on $k$ can be decreased [4] to $\mathcal{O}\left(k \cdot 2^{k}\right)$. As $k$ depends on the query in our case, and we are not concerned with query complexity in this paper, we do not explore such optimizations.

First, we define a variant of the permanent which is easier to analyze here. Given a matrix $M$ with rows $R$ and columns $C$, where $R$ and $C$ are both totally ordered, define $\operatorname{perm}^{\prime}(M)$ by the same expression as the permanent, but where $f$ only ranges over increasing functions $f: R \rightarrow C$. Fix an ordering of $C$. Then, $\operatorname{perm}(M)=\sum_{<_{R}} \operatorname{perm}^{\prime}(M)$, where $<_{R}$ ranges over all $|R|$ ! orderings of $R$. Since $|R|$ is considered fixed, it suffices to produce circuits computing perm' $(M)$.

Suppose $M$ is a $k \times n$ matrix, and let $1 \leqslant l \leqslant n$. By $A_{i}^{l}$ denote the submatrix of $M$ with rows $\{1, \ldots, i\}$ and columns $\{1, \ldots, l\}$, and by $B_{i}^{l}$ denote the submatrix with rows $\{i+1, \ldots, k\}$ and columns $\{l+1, \ldots, n\}$. The following lemma follows by grouping increasing functions $f:[k] \rightarrow[n]$ according to the moment they exceed $l$, i.e., $\max \{0 \leqslant i \leqslant$ $k: f(i) \leqslant l\}$.

Lemma 10. For a $k \times n$ matrix $M$ and $1 \leqslant l \leqslant n$, the following identity holds:

$$
\operatorname{perm}^{\prime}(M)=\sum_{i=0}^{k} \operatorname{perm}^{\prime}\left(A_{i}^{l}\right) \cdot \operatorname{perm}^{\prime}\left(B_{i}^{l}\right)
$$

Note that if $l=\lfloor n / 2\rfloor$, all the matrices involved in the sum have roughly half the number of columns as $M$. Recursively expanding the permanents on the right-hand side, we obtain a term which - viewed as a circuit - has the properties expressed in the following lemma.

Lemma 11. Fix a semiring $\mathbf{S}$ and a number $k \in \mathbb{N}$. Then there is a family $\left(C_{n}\right)_{n \in \mathbb{N}}$ of circuits without permanent gates computing the permanent of $k \times n$ matrices, and with depth $\mathcal{O}_{k}(\log n)$, fan-out $\mathcal{O}_{k}(1)$, reach-out $\mathcal{O}_{k}(\log n)$, and where each circuit $C_{n}$ is computable in time $\mathcal{O}_{k}(n)$.

This immediately yields the following. 
Corollary 12. The circuits $C_{\mathcal{A}}$ in Theorem 6 can be assumed to have no permanent gates, but logarithmic reach-out instead of bounded depth.

Note that a circuit $C$ with reach-out $c$ yields a dynamic data structure which allows maintaining the value $C(v)$ under updates modifying the valuation $v$ in time $\mathcal{O}(c)$. This yields the following:

Corollary 13. There is an algorithm which, given a weighted structure $\mathcal{A}(\mathbf{w})$ with $\mathcal{A} \in \mathscr{C}$, computes in time $\mathcal{O}_{\mathscr{C}, f}(|\mathcal{A}|)$ a dynamic data structure maintaining the value $f_{\mathcal{A}(\mathbf{w})}$ which allows to update $\mathbf{w}$ in time $\mathcal{O}_{\mathscr{C}, f}(\log |\mathcal{A}|)$.

Note that for the semirings $(\mathbb{N} \cup\{+\infty\}$, min, max $)$ or $(\mathbb{N} \cup\{+\infty\}$, min, +$)$, the permanent of a $1 \times n$ matrix is just the smallest entry of the matrix, and maintaining the smallest entry allows to implement sorting in the comparison model. This leads to the following.

Proposition 14. The logarithmic update time in Corollary 13 and Theorem 8 is tight, i.e., it cannot be improved for general semirings $\mathbf{S}$ while keeping the initialization time linear.

Therefore, to obtain a dynamic algorithm with linear initialization time and sublogarithmic update time, we need to assume some additional properties of the semiring $\mathbf{S}$.

\section{Rings}

The next lemma states that for rings, permanent gates can be completely eliminated.

Lemma $15(*)$. Suppose that $\mathbf{S}$ is a ring and fix a number $k \in \mathbb{N}$. Then there is a family $\left(C_{n}\right)_{n \in \mathbb{N}}$ of $\mathbf{S}$-circuits computing the permanent of $k \times n$ matrices, which has bounded depth, bounded fan-out, and where each circuit $C_{n}$ is computable in time $\mathcal{O}_{k}(n)$.

For example, for $k=2$, the permanent of a matrix with two rows $\left(a_{1} \ldots a_{n}\right)$ and $\left(b_{1} \ldots b_{n}\right)$ can be expressed as follows:

$$
\sum_{i \neq j} a_{i} b_{j}=\sum_{i} a_{i} \cdot \sum_{i} b_{i}-\sum_{i} a_{i} \cdot b_{i}
$$

and the right-hand side readily yields a term using only addition and multiplication, possibly by -1 . In general, we use an inclusion-exclusion formula.

Corollary 16. If in Theorem 6 we consider rings only, then the obtained circuits $C_{\mathcal{A}}$ have no permanent gates.

Note that a circuit $C$ with fan-out $c$ and constant depth $d$ has reach-out $\mathcal{O}\left(c^{d+1}\right)$, yielding constant-time updates:

Corollary 17. There is an algorithm which, given a weighted structure $\mathcal{A}(\mathbf{w})$ with $\mathcal{A} \in \mathscr{C}$ and weight functions $\mathbf{w}$ taking values in a ring $\mathbf{S}$, computes in linear time a dynamic data structure maintaining the value $f_{\mathcal{A}(\mathbf{w})}$ which allows to update $\mathbf{w}$ in constant time. 


\section{Finite semirings}

Another case where we can replace permanent gates by simpler circuits is the case of finite semirings. For those semirings, we replace permanent gates by circuits with counting gates: threshold gates and mod gates (the precise definition is in the appendix). The key observation is that the permanent of a $k \times n$ matrix $M$ can be computed based on the number of occurrences of each tuple $c \in \mathbf{S}^{k}$ as a column in $M$. This leads to the following lemma.

Lemma $18(*)$. Fix a finite semiring $\mathbf{S}$ and a number $k \in \mathbb{N}$. There is a family $\left(C_{n}\right)_{n \in \mathbb{N}}$ of $\mathbf{S}$-circuits with counting gates computing the permanent of $k \times n$ matrices, which has bounded depth, bounded fan-out, and each circuit $C_{n}$ is computable in time $\mathcal{O}_{k}(n)$, has thresholds bounded by $\mathcal{O}(|\mathbf{S}|+k)$, and the modulus of each mod gate is the order of a cyclic subgroup of $(\mathbf{S},+)$.

Corollary 19. If $\mathbf{S}$ is a finite semiring, then the circuits $C_{\mathcal{A}}$ in Theorem 6 can be assumed to be $\mathbf{S}$-circuits with counting gates with thresholds at most $\mathcal{O}_{f}(|\mathbf{S}|)$ and modulus as above. In particular, for the boolean semiring $\mathbf{B}$, the mod gates are absent.

If we assume a computation model which allows incrementing and decrementing integers, as well as testing divisibility by any fixed number, in constant time, then we get the following.

Corollary 20. There is an algorithm which, given a weighted structure $\mathcal{A}(\mathbf{w})$ with $\mathcal{A} \in \mathscr{C}$, computes in linear time a dynamic data structure maintaining the value $f_{\mathcal{A}(\mathbf{w})}$ which allows to update $\mathbf{w}$ in constant time.

Proof ( OF Theorem 8). The case when $f$ has no free variables follows from Corollaries 13,17 , and 20. The case of an expression $f(\mathbf{x})$ with free variables $\mathbf{x}=\left\{x_{1}, \ldots, x_{k}\right\}$ reduces to that case, by considering the closed expression

$$
f^{\prime}=\sum_{\mathbf{x}} f(\mathbf{x}) \cdot v_{1}\left(x_{1}\right) \cdots v_{k}\left(x_{k}\right),
$$

where $v_{1}, \ldots, v_{k}$ are new unary weight symbols, set to 0 by default. Then $f_{\mathcal{A}(\mathbf{w})}(\mathbf{a})=f_{\mathcal{A}(\mathbf{w v})}^{\prime}$ where for $i=1, \ldots, k$, the function $v_{i}$ maps $a_{i}$ to 1 and all other elements to 0 . Hence, querying the value of $f$ at a tuple a can be simulated by $2|\mathbf{x}|$ updates, as it amounts to temporarily setting the weights $v_{1}\left(a_{1}\right), \ldots, v_{k}\left(a_{k}\right)$ to 1 , querying the value of $f^{\prime}$, and then setting the weights back to 0 .

We remark that for some semirings, simulating querying by a sequence of updates may not be optimal. For example, if $\mathbf{S}=(\mathbb{N} \cup\{+\infty\}$, min, +$)$, then querying can be achieved in constant time, while still having logarithmic time updates and linear time initialization, even though updating provably requires logarithmic time, due to Proposition 14. To achieve 
these improved query times, again it is sufficient to solve the problem for the case of a permanent, where querying amounts to computing the permanent of the current matrix with a fixed number of values temporarily modified. For semirings such as above, constanttime querying can be achieved using heaps. We omit the details.

\section{Provenance semiring}

Provenance analysis is about tracing how a given query output was produced, and what are the properties of the computation which lead to this output. For example, one could try to analyze which input tuples are responsible for the fact that a given tuple is produced in the output, or what is the probability that a given output is produced, basing on some probability distribution on the inputs. The algebraic theory of provenance is based on semirings. Indeed, many instances of provenance analysis can be seen as evaluating queries in fixed semirings. It is known that the free semiring, considered below, is in a certain precise sense the most general semiring which can be used for provenance analysis [9].

The free semiring. Let $A$ be a (possibly infinite) set of symbols. The free (commutative) semiring generated by $A$, denoted $\mathbf{F}_{A}$, is the semiring consisting of sums of unordered sequences of elements of $A$, with addition and multiplication defined naturally. The 0 of this semiring is the empty sum, and the 1 is the sum with one summand, being the empty sequence. This semiring is isomorphic to the semiring of polynomials with variables from $A$ and coefficients from $\mathbb{N}$. It is also called the provenance semiring [9].

Example 21. Let $\Sigma$ be a signature consisting of a binary relation symbol $E$, and consider the query

$$
\varphi(x)=\exists_{y, z} E(x, y) \wedge E(y, z) \wedge E(z, x) .
$$

For the purpose of tracking provenance, consider the expression

$$
f(x)=\sum_{y, z} w(x, y) \cdot w(y, z) \cdot w(z, x) .
$$

Suppose $G=(V, E)$ is the directed graph with vertices $a, b, c, d$ and edges $a b, b c, c a, b d, d a$. Then, $\varphi_{G}(a)$ holds, and evaluating $f(a)$ in $G(w)$, where $w_{G}(x, y)=e_{x y}$ is a unique identifier for each $(x, y) \in E$, reveals why it holds:

$$
f_{\mathcal{A}}(a)=e_{a b} e_{b c} e_{c a}+e_{a b} e_{b d} e_{d a} .
$$


Iterators. As the elements of the free semiring be sums of a number of summands which depends on the data, we cannot reasonably assume that such elements can be represented in single memory cells. We therefore assume that each element of the free semiring is represented by an iterator, as follows.

A bi-directional iterator for a list $u_{1}, \ldots, u_{l}$ stores an index $i \in\{0,1, \ldots, l\}$, initially set to 1 , and implements the following methods:

current: output $u_{i}$, or $\perp$ if $i=0$.

next: increment $i$, modulo $l$,

previous: decrement $i$, modulo $l$,

We say that such a bi-directional iterator has access time $t$, where $t \in \mathbb{N}$, if the operations next, previous take time $t$, and the operation current, takes time $t \cdot\left|u_{i}\right|$, where $\left|u_{i}\right|$ is the length of $u_{i}$.

In the theorem below, for a given $\Sigma(\mathbf{w})$-structure $\mathcal{A}, \mathbf{w}_{\mathcal{A}}$ is a valuation in the semiring $\mathbf{F}_{A}$, given by providing a bi-directional iterator representing $w_{\mathcal{A}}(a)$, for each $a \in \mathcal{A}$ and $w \in \mathbf{w}$. An update to $\mathbf{w}$ modifies a single value $w_{\mathcal{A}}(a)$, by providing an iterator for the new value.

Theorem $22(*)$. Let $\mathscr{C}$ be a class of $\Sigma$-structures of bounded expansion and let $f(\mathbf{x})$ be a $\Sigma(\mathbf{w})$-expression. There is an algorithm which, given a structure $\mathcal{A} \in \mathscr{C}$ and a tuple $\mathbf{w}$ of weight functions with values in $\mathbf{F}_{A}$, where for each $w \in \mathbf{w}$ and $a \in \mathcal{A}$, the element $w_{\mathcal{A}}(a)$ is represented by a bi-directional iterator with access time bounded by a constant, computes in linear time a data structure which allows to query any tuple $\mathbf{a} \in \mathcal{A}^{\mathbf{x}}$ and obtain in constant time a bi-directional iterator for $f_{\mathcal{A}(\mathbf{w})}(\mathbf{a})$ with constant access time. The data structure is maintained in constant time upon updates to $\mathbf{w}$.

Note that the element $f_{\mathcal{A}(\mathbf{w})}(\mathbf{a})$ of $\mathbf{F}_{A}$ may have repeating summands, and the enumerator will enumerate each repetition separately. This will not be a problem when we apply the result above in Theorem 24 later to enumerate all answers to a first-order query without repetitions.

To prove Theorem 22, we apply our circuit framework and Theorem 6 in particular, which essentially tell us that we only need to consider the case of permanents, handled below:

Lemma 23 (*). There is a dynamic algorithm which, given an $R \times C$ matrix $M$ with entries from $\mathbf{F}_{A}$, where each entry $M[r, c]$ is represented by a bi-directional iterator with access time $t$, computes in time $\mathcal{O}_{R}(C)$ a data structure which maintains a bi-directional iterator for $\operatorname{perm}(M)$ with access time $\mathcal{O}_{R}(t)$, and updates it in constant time, whenever an entry of $M$ is updated. 


\section{Dynamic query enumeration}

We now apply our framework to yield a dynamic algorithm for enumerating the answers $\varphi_{\mathcal{A}}$ to a first-order query $\varphi(\mathbf{x})$. Below, we consider updates to a $\mathcal{A}$ structure which can add/remove single tuples to its relations provided that they preserve the Gaifman graph. This amounts to saying that a tuple a can be added to a relation only if its elements form a clique in the Gaifman graph of $\mathcal{A}$.

Theorem $24(*)$. Let $\Sigma$ be a signature, let $\varphi(\mathbf{x})$ be a first-order $\Sigma$-formula, and let $\mathscr{C}$ be a class of $\Sigma$-structures of bounded expansion. There is an algorithm which inputs a structure $\mathcal{A} \in \mathscr{C}$ and computes in linear time a dynamic data structure which provides a constant access, bi-directional iterator for $\varphi_{\mathcal{A}}$. The data structure is maintained in constant time upon updates to $\mathcal{A}$ which preserve the Gaifman graph.

Note that the static version of Theorem 24, without the last part, is the result of Kazana and Segoufin [14].

Proof (Sketch For Theorem 24). We sketch the proof in the static case, thus reproving the main result of [14].

By Theorem 3 it is enough to consider the case when $\varphi(\mathbf{x})$ is quantifier-free. Fix an input structure $\mathcal{A}$ and an enumeration $\mathbf{x}=\left\{x_{1}, \ldots, x_{k}\right\}$. Let $\mathbf{F}$ be the free semiring generated by identifiers of the form $e_{a}^{i}$, where $i \in\{1, \ldots, k\}$ and $a \in \mathcal{A}$. Its elements are expressions such as $e_{a}^{1} e_{b}^{2} e_{c}^{3}+e_{b}^{1} e_{c}^{2} e_{d}^{3}$. For each $i$ with $1 \leqslant i \leqslant k$, define an $\mathbf{F}$-valued weighted function $w_{i}$ on $\mathcal{A}$, where $w_{i}(a)=e_{a}^{i} \in \mathbf{F}$ for $a \in \mathcal{A}$. Note that for $a_{1}, \ldots, a_{k} \in \mathcal{A}$, the expression $w_{1}\left(a_{1}\right) \cdots w_{k}\left(a_{k}\right)$ evaluates in the structure $\mathcal{A}(\mathbf{w})$ to the element $e_{a_{1}}^{1} \cdots e_{a_{k}}^{k}$ of $\mathbf{F}$, representing the tuple $\mathbf{a} \in \mathcal{A}^{\mathbf{x}}$ with $\mathbf{a}\left[x_{i}\right]=a_{i}$. Consider the $\Sigma(\mathbf{w})$-expression:

$$
f=\sum_{\mathbf{x}}[\varphi(\mathbf{x})] \cdot w_{1}\left(x_{1}\right) \cdots w_{k}\left(x_{k}\right)
$$

Then $f_{\mathcal{A}(\mathbf{w})}$ is the element of $\mathbf{F}$ representing the set of tuples $\varphi_{\mathcal{A}} \subseteq \mathcal{A}^{\mathbf{x}}$, with one occurrence per each tuple. Note that each weight $w_{i}(a)$ is a single element $e_{a}^{i} \in \mathbf{F}$, so can be trivially represented by a bi-directional iterator with constant access time. Applying Theorem 22 yields a bi-directional iterator with constant access time, which iterates through all the summands of the element $f_{\mathcal{A}(\mathbf{w})}$, corresponding to the tuples in $\varphi_{\mathcal{A}}$.

The dynamic case proceeds similarly, but instead of Theorem 3, uses its dynamic version [7, Theorem 6.3] to reduce to the case where $\varphi(\mathbf{x})$ is quantifier-free. We then maintain the representation of each relation symbol $R$ and its negation as a weight function with values in $\{0,1\} \subseteq \mathbf{F}$, and replace the expression $[\varphi(\mathbf{x})]$ in (4) by an equivalent expression involving those weight functions. Finally, we utilize the dynamic data structure given by Theorem 22 to maintain an enumerator for $f_{\mathcal{A}}(\mathbf{w})$. The details are in the appendix. 
Theorem 24 only allows updates which preserve the Gaifman graph of the input structure. This includes, in particular, updates which only modify unary predicates. The following example ${ }^{2}$ shows that already this yields a useful data structure.

Example 25. A local search algorithm searches for an optimal solution - e.g. largest independent set or smallest dominating set in a given graph - by iteratively trying to improve the the current solution locally, i.e., by modifying in each round the current solution by adding/removing at most $\lambda$ vertices, for some fixed locality radius $\lambda$. In case of independent set or dominating set, the current solution can be represented by a unary predicate, and a fixed first-order formula (depending on $\lambda$ ) can determine the existence of a local improvement. Moreover, using enumeration, we can find any such improvement in constant time, and then use it to improve the current solution by performing a constant number of updates, in constant time. Hence, for graphs from a fixed class of bounded expansion, using the data structure provided by Theorem 24, each round of the local search algorithm can be performed in constant time, and the local search algorithm will compute a local optimum in linear time.

This observation can be combined with a recent result of Har-Peled and Quanrud [13], stating that local search with sufficiently large radius $\lambda$ yields a $(1+\varepsilon)$-approximation for these problems on any fixed class of graphs with polynomial expansion, where $\lambda$ depends only on $\varepsilon, r$, and the class. The definition of a polynomial expansion class is obtained by requiring that the function $r \mapsto c_{r}$ in the definition of a bounded expansion class is polynomial. These classes include e.g. the class of planar graphs. This yields efficient lineartime approximation schemes (in particular, EPTASes) for the DISTANCE- $r$ INDEPENDENT Set and Distance- $r$ Dominating Set problems on any class of graphs with polynomial expansion. This improves the result of [13], which only obtains a PTAS.

\section{$7 \quad$ Nested weighted query evaluation}

We now introduce nested weighted queries which can handle multiple semirings. They involve a slightly different syntax than the one used previously.

If $A$ is a set and $\mathbf{S}$ is a semiring, then an $\mathbf{S}$-relation of arity $k$ on $A$ is a function $R: A^{k} \rightarrow \mathbf{S}$. We consider structures $\mathcal{A}$ equipped with relations with values in various semirings. Classical relational structures are recovered by only allowing relations with values in the boolean semiring. Formulas can be constructed using semiring summation $\sum$ playing the role of quantification, and semiring operations + and · playing the role of connectives. Additionally we allow other connectives which can transfer between semirings. This is defined below.

Connectives. Let $\mathbb{C}$ be a collection of semirings and of connectives which are functions $c: \mathbf{S}_{1} \times \cdots \times \mathbf{S}_{k} \rightarrow \mathbf{S}$, where $\mathbf{S}, \mathbf{S}_{1}, \ldots, \mathbf{S}_{k} \in \mathbb{C}$ are semirings. Examples of connectives

\footnotetext{
${ }^{2}$ suggested by Zdeněk Dvořák and also Felix Reidl, Micha Pilipczuk, Sebastian Siebertz
} 
include the function $<: \mathbf{N} \times \mathbf{N} \rightarrow \mathbf{B}$, corresponding to the total order on $\mathbf{N}$, or the function $/: \mathbf{Q} \times \mathbf{Q} \rightarrow \mathbf{Q}$, where $\mathbf{Q}$ is the field of rationals, which maps a pair $p, q$ to $\frac{p}{q}$ if $q \neq 0$ and to 0 otherwise. Another example is the Iverson bracket denoted $[\cdot]_{\mathbf{S}}: \mathbf{B} \rightarrow \mathbf{S}$, where $\mathbf{S}$ is a semiring, mapping $0 \in \mathbf{B}$ to $0 \in \mathbf{S}$ and $1 \in \mathbf{B}$ to $1 \in \mathbf{S}$.

Signatures, structures, and Gaifman graphs. A $\mathbb{C}$-signature $\Sigma$ is a family of $\mathbf{S}$ relation symbols, for each semiring $\mathbf{S} \in \mathbb{C}$, and function symbols, each with a specified arity. A $\Sigma$-structure $\mathcal{A}$ is a set $A$ together with a function $f_{\mathcal{A}}: A^{k} \rightarrow A$ for each function symbol $f \in \Sigma$ of arity $k$, and together with an $\mathbf{S}$-relation $R_{\mathcal{A}}: A^{k} \rightarrow \mathbf{S}$ for each $\mathbf{S}$-relation symbol $R \in \Sigma$ of arity $k$. If $\Sigma$ contains no function symbols, then the Gaifman graph of a $\Sigma$-structure $\mathcal{A}$ is the graph whose vertices are the elements of $\mathcal{A}$, and where two distinct elements $v, w$ are adjacent if there is some $R \in \Sigma$ and some tuple $\mathbf{a} \in R_{\mathcal{A}}$ such that $R(\mathbf{a}) \neq 0$ and a contains $v$ and $w$. As usual, we may convert a structure with function symbols into a structure which only uses relation symbols, by interpreting a function $f: \mathcal{A}^{k} \rightarrow \mathcal{A}$ as a B-relation $R: \mathcal{A}^{k+1} \rightarrow \mathbf{B}$ representing the graph of $f$. Via this conversion, we define the Gaifman graph of a structure with function symbols. A class of $\Sigma$-structures has bounded expansion if the class of its Gaifman graphs has this property.

The logic $\mathrm{FO}[\mathbb{C}] \quad$ Fix a set $\mathbb{C}$ of semirings and connectives, and a $\mathbb{C}$-signature $\Sigma$. We implicitly assume that $\mathbb{C}$ contains the boolean semiring $\mathbf{B}$ and that $\Sigma$ contains the binary equality symbol = as a binary B-relation symbol.

We define the syntax of the logic $\mathrm{FO}[\mathbb{C}]$. Each formula $\varphi$ has a specified output type, which is a semiring $\mathbf{S} \in \mathbb{C}$. If the output type is $\mathbf{S}$, then we say that $\varphi$ is an $\mathbf{S}$-valued formula. The set of $\mathbf{S}$-valued $\mathrm{FO}[\mathbb{C}]$-formulas is defined inductively, and consists of the following formulas:

- $R\left(t^{1}, \ldots, t^{k}\right)$, where $R \in \Sigma$ is an $\mathbf{S}$-relation symbol of arity $k$ and $t^{1}, \ldots, t^{k}$ are terms built out of variables and function symbols from $\Sigma$.

- $s$, where $s \in \mathbf{S}$ is treated as a constant.

- $c\left(\varphi^{1}, \ldots, \varphi^{k}\right)$, where $\varphi^{1}, \ldots, \varphi^{k}$ are $\mathrm{FO}[\mathbb{C}]$-formulas with output types $\mathbf{S}_{1}, \ldots, \mathbf{S}_{k}$, respectively, and $c: \mathbf{S}_{1} \times \cdots \times \mathbf{S}_{k} \rightarrow \mathbf{S}$ is a connective in $\mathbb{C}$.

- $\sum_{x} \varphi$, where $\varphi$ is an $\mathbf{S}$-valued $\mathrm{FO}[\mathbb{C}]$-formula.

- $\varphi_{1}+\varphi_{2}$ and $\varphi_{1} \cdot \varphi_{2}$, where $\varphi_{1}$ and $\varphi_{2}$ are $\mathbf{S}$-valued $\mathrm{FO}[\mathbb{C}]$-formulas.

- $[\varphi]_{\mathbf{S}}$, where $\varphi$ is a $\mathbf{B}$-valued $\mathrm{FO}[\mathbb{C}]$-formula.

- $\neg \varphi$, if $\mathbf{S}=\mathbf{B}$ and $\varphi$ is a $\mathbf{B}$-valued FO[C]-formula. 
For a $\mathbf{B}$-valued formula $\varphi$ in $\mathrm{FO}[\mathbb{C}]$ we write $\vee$ and $\wedge$ instead of + and $\cdot$, respectively, $\exists_{x} \varphi$ instead of $\sum_{x} \varphi$, and $\forall_{x} \varphi$ as syntactic sugar for $\neg \exists_{x} \neg \varphi$. Note that first-order logic coincides with $\mathrm{FO}[\mathbb{C}]$ when $\mathbb{C}$ contains only the boolean semiring $\mathbf{B}$.

We write $\varphi(\mathbf{x})$ to indicate that $\varphi$ has free variables contained in $\mathbf{x}$. The semantics of an $\mathbf{S}$-valued formula $\varphi(\mathbf{x})$ in a given structure $\mathcal{A}$ is a function $\varphi_{\mathcal{A}}: \mathcal{A}^{\mathbf{x}} \rightarrow \mathbf{S}$, and is defined inductively, as expected.

Counting logics. The papers $[15,12]$ study a logic denoted $\operatorname{FOC}(\mathbb{P})$, where $\mathbb{P}$ is a set of numerical predicates of the form $\mathrm{P}: \mathbb{Z}^{k} \rightarrow \mathbf{B}$. This logic can be seen as the fragment of our logic $\mathrm{FO}[\mathbb{P} \cup\{\mathbf{B}, \mathbb{Z}\}]$, where summation in $\mathbb{Z}$ is restricted to counting terms of the form $\#_{\mathbf{x}} \varphi$, in our syntax corresponding to $\sum_{\mathbf{x}}[\varphi]_{\mathbb{Z}}$. As observed by Grohe and Schweikardt [12], the problem of evaluation of $\mathrm{FOC}(\mathbb{P})$ on the class of trees is as hard as the problem of evaluation of first-order logic on arbitrary graphs. In particular, under common complexity-theoretic assumptions, there is no algorithm which decides whether a given sentence $\varphi \in \operatorname{FOC}(\mathbb{P})$ holds in a given tree $T$, whose running time is $\mathcal{O}\left(|T|^{c}\right)$, where $c \in \mathbb{N}$ is independent of $\varphi$. Due to this, Grohe and Schweikardt propose to study a restricted fragment of the logic $\mathrm{FOC}(\mathbb{P})$, denoted $\mathrm{FOC}_{1}(\mathbb{P})$. In this fragment, numerical predicates in $\mathbb{P}$ can be applied only if they yield a formula with at most one free variable. So for example, $\mathrm{P}(\varphi(x), \psi(y))$ is no longer allowed as a formula, but $\mathrm{P}(\varphi(x), \psi(x))$ is. Grohe and Schweikardt proved that model-checking of $\mathrm{FOC}_{1}(\mathbb{P})$ has an almost-linear time algorithm on nowhere dense classes.

The restricted fragment $\mathrm{FO}_{\mathrm{G}}[\mathbb{C}]$. We introduce an analogous fragment of $\mathrm{FO}[\mathbb{C}]$, in which the following restriction is imposed on how connectives $c \in \mathbb{C}$ can be used. The syntax is the same as for $\mathrm{FO}[\mathbb{C}]$, with the difference that the construct $c\left(\varphi^{1}, \ldots, \varphi^{k}\right)$ is replaced by $\left[R\left(x_{1}, \ldots, x_{l}\right)\right]_{\mathbf{S}} \cdot c\left(\varphi^{1}, \ldots, \varphi^{k}\right)$ where in the guard $\left[R\left(x_{1}, \ldots, x_{l}\right)\right]_{\mathbf{S}}$, the symbol $R$ is a B-relation symbol in $\Sigma$ and $\left\{x_{1}, \ldots, x_{l}\right\}$ contains all the free variables of $\varphi^{1}, \ldots, \varphi^{k}$. For example, all formulas discussed in the introduction, as well as $\sum_{x} \sum_{y}[E(x, y)]_{\mathbb{Q}}$. weight $(x) /$ weight $(y)$, are in $\mathrm{FO}_{\mathrm{G}}[\mathbb{C}]$, whereas the formula $\sum_{x} \sum_{y}$ weight $(x) /$ weight $(y)$ is not (here $/: \mathbb{Q} \times \mathbb{Q} \rightarrow \mathbb{Q}$ is a connective).

Note that $\mathrm{FO}_{\mathrm{G}}[\mathbb{C}]$ still extends first-order logic, and also $\mathrm{FAQ}$ queries over one semiring (without product aggregates). Also, if $\mathbb{P}$ is a set of numerical predicates, then $\mathrm{FO}_{\mathrm{G}}[\mathbb{P} \cup\{\mathbf{B}, \mathbb{Z}\}]$ strictly extends the logic $\mathrm{FOC}_{1}(\mathbb{P})$ studied in [12], as it allows unrestricted summation over $\mathbb{Z}$, rather than counting.

The main result of this paper is the following:

Theorem $26(*)$. Let $\mathbb{C}$ be a collection of semirings and connectives, in which all operations take constant time, let $\Sigma$ be a $\mathbb{C}$-signature, let $\mathscr{C}$ be a class of $\Sigma$-structures of bounded expansion, and let $\varphi(\mathbf{x}) \in \mathrm{FO}_{\mathrm{G}}[\mathbb{C}]$ be a $\Sigma$-formula.

There is an algorithm which, given a structure $\mathcal{A} \in \mathscr{C}$, computes a data structure allowing to query the value $\varphi_{\mathcal{A}}(\mathbf{a})$, given a tuple $\mathbf{a} \in \mathcal{A}^{\mathbf{x}}$. The algorithm runs in time $\mathcal{O}_{\varphi}(|\mathcal{A}| \log |\mathcal{A}|)$ in general, and in time $\mathcal{O}_{\varphi}(|\mathcal{A}|)$ if all the semirings in $\mathbb{C}$ are either rings or 
finite. The query time is $\mathcal{O}_{\varphi}(\log |\mathcal{A}|)$ in general, and $\mathcal{O}_{\varphi}(1)$ if the output semiring of $\varphi$ is either a ring or is finite.

Moreover, $\varphi(\mathbf{x})$ has output in the boolean semiring, then the data structure also provides a constant-delay enumerator for $\varphi_{\mathcal{A}}$.

Proof (SketCh). To prove the theorem, we proceed by induction on the size of the formula $\varphi(\mathbf{x}) \in \mathrm{FO}_{\mathrm{G}}[\mathbb{C}]$. In the inductive step, replace every guarded connective $\left[R\left(x_{1}, \ldots, x_{l}\right)\right]$. $c\left(\varphi^{1}, \ldots, \varphi^{k}\right)$ occurring in $\varphi$ at the top-most level (i.e. not within the scope of another connective) by a new weight symbol $r\left(x_{1}, \ldots, x_{l}\right)$. This yields a formula with no connectives, which can be viewed as a weighted expression $f$ in the sense of Section 3, or a boolean formula if $\varphi$ is $\mathbf{B}$-valued. Compute the new weights $r_{\mathcal{A}}\left(x_{1}, \ldots, x_{l}\right)=\left[R_{\mathcal{A}}\left(x_{1}, \ldots, x_{l}\right)\right]$.

$c\left(\varphi_{\mathcal{A}}^{1}, \ldots, \varphi_{\mathcal{A}}^{k}\right)$ in $\mathcal{A}$ using the inductive assumption applied to $\varphi^{1}, \ldots, \varphi^{k}$, and then evaluate $f$ with the newly computed weights, using Theorem 8 , or Theorem 3 if $\varphi$ is $\mathbf{B}$-valued. For the last part, if $\varphi(\mathbf{x})$ has output in the boolean semiring, we apply Theorem 24 to the boolean formula $f(\mathbf{x})$ considered above, yielding a constant-delay enumerator for $\varphi_{\mathcal{A}}$.

\section{Conclusion}

We presented an algebraic framework which allows to derive some existing and some new results concerning the evaluation, enumeration, and maintenance of query answers on sparse databases. The main advantage of our framework is that it allows to achieve new, efficient algorithms, by simple algebraic considerations about the permanent in various semirings. Proving those results directly would be considerably more involved. In particular, Theorem 26 provides an efficient algorithm for enumerating answers to queries from a complex query language involving aggregates from multiple semirings. Our results partially resolve two questions posed in [12, Section 9]: we can answer aggregate queries with the usual SQL aggregates, and we can enumerate the answers to such queries, for classes of bounded expansion.

In this paper, we only study the basic applications of the main technical result, Theorem 6 . We believe that following these principles will allow to extend the results in further directions, perhaps by considering more intricate semirings and properties of their permanents. For example, extending Theorem 26 to handle updates will require analyzing the interaction between the allowed connectives and the semiring operations. Furthermore, in our dynamic enumeration algorithm, Theorem 24, we only handle updates which preserve the Gaifman graph. Arbitrary updates could be handled if we could have a dynamic algorithm for maintaining a low treedepth decomposition of the Gaifman graph. Such an algorithm would immediately allow to lift Theorem 24 to arbitrary updates which leave the structure in the considered class of databases $\mathscr{C}$. For some graph classes $\mathscr{C}$, such as classes of bounded degree or bounded treewidth, such an algorithm can be trivially obtained. This allows, e.g., to derive the result of [3] (without mod quantifiers) from Theorem 24. 
Another question which arises is about generalizing Theorem 6 to other graph classes and logics. In particular - to nowhere dense graph classes, which are more general than bounded expansion classes, and to classes of bounded treewidth, which are less general, but come with the more powerful MSO logic. For nowhere dense classes, no analogue of the quantifier elimination result, Theorem 3 is known. Our main technical result, Theorem 6, is closely related to quantifier-elimination. Indeed, it is not difficult to derive Theorem 3 from the quantifier-free case of Theorem 6 applied in the boolean semiring (where summation still allows introducing existential quantifiers). Hence, generalizing Theorem 6 to nowhere dense classes is closely connected to obtaining a quantifier elimination procedure there.

\section{References}

[1] A. Amarilli, P. Bourhis, L. Jachiet, and S. Mengel. A circuit-based approach to efficient enumeration. In ICALP 2017, pages 111:1-111:15, 2017.

[2] A. Amarilli, P. Bourhis, and S. Mengel. Enumeration on trees under relabelings. In ICDT 2018, pages 5:1-5:18, 2018.

[3] C. Berkholz, J. Keppeler, and N. Schweikardt. Answering FO+MOD queries under updates on bounded degree databases. ACM Trans. Database Syst., 43(2):7:1-7:32, 2018 .

[4] A. Björklund, T. Husfeldt, P. Kaski, and M. Koivisto. Evaluation of permanents in rings and semirings. Inf. Process. Lett., 110(20):867-870, Sept. 2010.

[5] A. Darwiche. Compiling knowledge into decomposable negation normal form. In IJCAI 99, pages 284-289, 1999.

[6] Z. Dvorák, D. Král, and R. Thomas. Deciding first-order properties for sparse graphs. In FOCS 2010, pages 133-142, 2010.

[7] Z. Dvořák, D. Král, and R. Thomas. Testing first-order properties for subclasses of sparse graphs. Journal of the ACM (JACM), 60(5):36, 2013.

[8] J. Gajarský, S. Kreutzer, J. Nešetřil, P. O. de Mendez, M. Pilipczuk, S. Siebertz, and S. Toruńczyk. First-order interpretations of bounded expansion classes. In ICALP 2018, pages 126:1-126:14, 2018.

[9] T. J. Green, G. Karvounarakis, and V. Tannen. Provenance semirings. In PODS 2007, pages $31-40,2007$.

[10] M. Grohe and S. Kreutzer. Methods for algorithmic meta theorems. Model Theoretic Methods in Finite Combinatorics, 558:181-206, 2011. 
[11] M. Grohe, S. Kreutzer, and S. Siebertz. Deciding first-order properties of nowhere dense graphs. In STOC 2014, pages 89-98. ACM, 2014.

[12] M. Grohe and N. Schweikardt. First-order query evaluation with cardinality conditions. In PODS 2018, pages 253-266, 2018.

[13] S. Har-Peled and K. Quanrud. Approximation algorithms for polynomial-expansion and low-density graphs. SIAM J. Comput., 46(6):1712-1744, 2017.

[14] W. Kazana and L. Segoufin. Enumeration of first-order queries on classes of structures with bounded expansion. In PODS 2013, pages 297-308, 2013.

[15] D. Kuske and N. Schweikardt. First-order logic with counting: At least, weak hanf normal forms always exist and can be computed! In LICS 2017, pages 73:1-73:12, 2017.

[16] J. Nešetrril and P. Ossona de Mendez. Grad and classes with bounded expansion I. Decompositions. European Journal of Combinatorics, 29(3):760-776, 2008.

[17] J. Nešetrril and P. Ossona de Mendez. Grad and classes with bounded expansion II. Algorithmic aspects. European Journal of Combinatorics, 29(3):777-791, 2008.

[18] J. Nešetřil and P. Ossona de Mendez. Sparsity - Graphs, Structures, and Algorithms, volume 28 of Algorithms and combinatorics. Springer, 2012.

[19] D. Olteanu and J. Závodný. Factorised representations of query results: Size bounds and readability. In ICDT 2012, pages 285-298, 2012.

[20] N. Schweikardt, L. Segoufin, and A. Vigny. Enumeration for FO queries over nowhere dense graphs. In PODS 2018, pages 151-163, 2018. 


\section{A Proof Theorem 6}

In this section we prove Theorem 6 . To this end, we need to describe, for each class $\mathscr{C}$ of bounded expansion and closed quantifier-free $\Sigma(\mathbf{w})$-expression $f$, a construction of a class of circuits with bounded expansion. This will be achieved by a composition of a series of steps, in each step considering more complicated classes $\mathscr{C}$.

\section{A.1 Proof outline}

To prove Theorem 6, we will show a series of reductions depicted in Figure 2, and prove that the statement of the theorem holds when $\mathscr{C}$ is a class of labelled forests of bounded depth. The notion of reduction used here is made formal below. The reductions depicted in the figure will be shown in subsequent sections.

A binary relational structure is a structure over a signature consisting of unary and binary relation symbols.

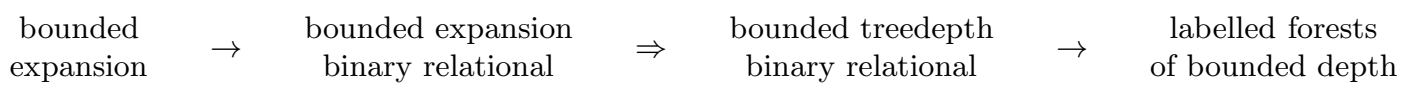

Figure 2: Outline of the proof of Theorem 6. An arrow $X \rightarrow Y$ signifies that every class of structures with property $X$ reduces to some class with property $Y . X \Rightarrow Y$ is a different kind of reduction, using the fact that classes of bounded expansion admit low-treedepth colorings.

Reductions. We say that a class $\mathscr{C}$ reduces to $\mathscr{D}$ if for every atomic formula $\alpha(\mathbf{x})$ of the form $f\left(x_{1}, \ldots, x_{k}\right)=y$ or $R\left(x_{1}, \ldots, x_{k}\right)$, where $x_{1}, \ldots, x_{k}, y$ are variables in $\mathbf{x}$ and $f$ and $R$ are function/relation symbols in the signature of $\mathscr{C}$, there is a quantifier-free formula $\alpha^{\prime}(\mathbf{x})$ in the signature of $\mathscr{D}$, and there is a linear algorithm which inputs a structure $\mathcal{A} \in \mathscr{C}$ and computes a structure $\mathcal{A}^{\prime} \in \mathscr{D}$, such that $\alpha_{\mathcal{A}}=\alpha_{\mathcal{A}^{\prime}}^{\prime}$ for all $\alpha$ as above.

Lemma 27. If $\mathscr{C}$ reduces to $\mathscr{D}$ and the statement of Theorem 6 holds for $\mathscr{D}$, then it holds for $\mathscr{C}$, where in both cases, we assume $f$ is quantifier-free and the arities of all weight functions in $\mathbf{w}$ to be 1 .

Before proving Lemma 27, we observe that it is enough to assume in the statement of Theorem 6 that the expression $f$ is simple, i.e.,

- for every expression $w(t)$ occurring in $f$, where $w \in \mathbf{w}$ and $t$ is a term, $t$ is a single variable,

- for every expression $[\alpha]$ occurring in $f, \alpha$ is a literal of the form $R\left(x_{1}, \ldots, x_{k}\right)$ or $\neg R\left(x_{1}, \ldots, x_{k}\right)$ for some relation symbol $R \in \Sigma \cup\{=\}$ or $f\left(x_{1}, x_{2}, \ldots, x_{k}\right)=y$ for some function symbol $f$, where $x_{1}, \ldots, x_{k}, y$ are variables. 
Lemma 28. Every $\Sigma(\mathbf{w})$-expression $f$ is equivalent to a simple expression $f^{\prime}$.

Proof. We apply a series of simplifications to $f$.

In the first step, we remove disjunctions and conjunctions from expressions $[\alpha]$ occurring in $f$. To this end, note that $[\alpha \wedge \beta]$ is equivalent to $[\alpha] \cdot[\beta]$, whereas $[\alpha \vee \beta]$ is equivalent to $[\alpha][\beta]+[\alpha][\neg \beta]+[\neg \alpha][\beta]$. Hence, we may assume that every expression occurring in $\alpha$ is a literal, i.e. an atom $R\left(t_{1}, \ldots, t_{k}\right)$ or its negation, where $R \in \Sigma \cup\{=\}$, and $t_{1}, \ldots, t_{k}$ are terms.

Next, observe that the expression $\left[R\left(t_{1}, \ldots, t_{k}\right)\right]$ is equivalent to the expression

$$
\sum_{x_{1}, \ldots, x_{k}}\left[t_{1}=x_{1}\right] \cdots\left[t_{k}=x_{k}\right]\left[R\left(x_{1}, \ldots, x_{k}\right)\right] \text {. }
$$

The same holds if $R$ is replaced by $\neg R$. Further, each expression $[t=x]$ as above can be iteratively simplified, if $t$ has nesting depth larger than 1 , as follows: if $t=f\left(t_{1}, \ldots, t_{k}\right)$ then $[t=x]$ is equivalent to $\sum_{y_{1}, \ldots, y_{k}}\left[y_{1}=t_{1}\right] \cdots\left[y_{k}=t_{k}\right][f(y)=x]$. Note that $t_{1}, \ldots, t_{k}$ have smaller depth than $t$.

After performing all these simplifications, we arrive at an expression $f$ where all boolean formulas $\alpha$ occurring in $f$ are of the form $R\left(x_{1}, \ldots, x_{k}\right), \neg R\left(x_{1}, \ldots, x_{k}\right)$, or $f\left(x_{1}, \ldots, x_{k}\right)=$ $y$.

Finally, each expression $w(t)$, where $w \in \mathbf{w}$ and $t$ is a term, can be replaced by $\sum_{x}[t=$ $x] \cdot w(x)$, and then $[t=x]$ can be simplified as described above.

Proof (of Lemma 27). Assume that the statement of Theorem 6 (for $f$ quantifier-free) holds for $\mathscr{D}$ and that $\mathscr{C}$ reduces to $\mathscr{D}$. We prove that the statement of Theorem 6 holds for $\mathscr{C}$ and $f$ quantifier-free.

Let $\Sigma$ be the signature of $\mathscr{C}$ and let $f$ be a closed $\Sigma(\mathbf{w})$-expression. By Lemma 28 we may assume that $f$ is simple. Define a $\Sigma^{\prime}(\mathbf{w})$-expression $f^{\prime}$ by replacing in $f$ each atomic boolean formula $\alpha$ by the equivalent $\Sigma^{\prime}$-formula $\alpha^{\prime}$, where $\alpha^{\prime}$ is as in the definition of a reduction. Then $f^{\prime}$ is quantifier-free and $f_{\mathcal{A}}=f_{\mathcal{A}^{\prime}}^{\prime}$, where $\mathcal{A}^{\prime}$ is the output of the algorithm as in the definition of a reduction.

Let $\left(D_{\mathcal{A}^{\prime}}\right)_{\mathcal{A}^{\prime} \in \mathscr{D}}$ be a linear-time computable family of circuits evaluating $f^{\prime}$ for the class $\mathscr{D}$. Given a structure $\mathcal{A} \in \mathscr{C}$, define the circuit $C_{\mathcal{A}}$ as $D_{\mathcal{A}}^{\prime}$, where $\mathcal{A}^{\prime}$ is computed by the algorithm from the reduction. Then $\left(C_{\mathcal{A}}\right)_{\mathcal{A} \in \mathscr{C}}$ is a linear-time computable family of circuits evaluating $f$ for the class $\mathscr{C}$.

\section{A.2 Forests of bounded depth}

In this section, we prove a special case of Theorem 6 , in the case when $\mathscr{C}$ is a class of labelled, rooted forests of bounded depth. We treat such a forest as a structure over a signature consisting of unary relation symbols for representing the labels, a unary relation symbol root which is interpreted in a rooted forest $F$ as the set of roots, and one unary 
function symbol parent, which is interpreted in a forest $F$ as the function mapping every non-root node to its parent, and the roots to themselves.

Lemma 29. The statement of Theorem 6 holds when $\mathscr{C}$ is a class of labeled forests of bounded depth, and $f$ is quantifier-free.

In the remainder of this section, fix a number $d \in \mathbb{N}$ and signature $\Sigma$ as above. By forest we mean a $\Sigma$-structures which is a forest, as described above. Fix a $\Sigma(\mathbf{w})$-expression $f$.

Basic expressions. A shape with free variables $\mathbf{x}$ is a forest $F$ and a tuple $\mathbf{a} \in F^{\mathbf{x}}$. We write $F(\mathbf{x})$ for the shape defined by $F$ and $\mathbf{a}$, and write $F[x]$ instead of $\mathbf{a}[x]$ for $x \in \mathbf{x}$, and $F[\mathbf{x}]$ for $\{F[x]: x \in \mathbf{x}\}$. We require that every node in a shape $F$ is an ancestor of some node in $F[\mathbf{x}]$. We distinguish shapes only up to isomorphism, i.e., we consider two shapes $F, G$ equal if there is a forest isomorphism from $F$ to $G$ which maps $F[x]$ to $G[x]$, for each $x \in \mathbf{x}$.

The atomic type of a shape $F$ is the set of all literals which hold in $F$. Specifically, those literals are:

$$
U\left(\text { parent }^{i}(x)\right)
$$

for $0 \leqslant i \leqslant d, x \in \mathbf{x}$, and unary predicate $U \in \Sigma$, such that $U\left(\right.$ parent $\left.^{i}(F[x])\right)$ holds,

$$
\operatorname{parent}^{i}(x)=\operatorname{parent}^{j}(y),
$$

for $0 \leqslant i, j \leqslant d$ and $x, y \in \mathbf{x}$ such that parent ${ }^{i}(F[x])=\operatorname{parent}^{j}(F[y])$

$$
\operatorname{parent}^{i}(x) \neq \operatorname{parent}^{j}(y),
$$

for $0 \leqslant i, j \leqslant d$ and $x, y \in \mathbf{x}$ such that $\operatorname{parent}^{i}(F[x]) \neq \operatorname{parent}^{j}(F[y])$.

With each shape $F(\mathbf{x})$ we associate the $\Sigma$-formula, also denoted $F(\mathbf{x})$, defined as the conjunction of all literals in the atomic type of $F$. The following is immediate.

Lemma 30. Given a forest $\mathcal{A}$ and a tuple $\mathbf{a} \in \mathcal{A}^{\mathbf{x}}$, the formula $F_{\mathcal{A}}(\mathbf{a})$ holds iff there is an embedding $\alpha: F \rightarrow \mathcal{A}$ of rooted forests which maps $F[x] \in F$ to $\mathbf{a}[x] \in \mathcal{A}$, for $x \in \mathbf{x}$. If such an embedding exists, it is unique.

A labelled shape is a pair $(F(\mathbf{x}), \lambda)$, where $F(\mathbf{x})$ is a shape and $\lambda$ associates to each node $v$ of $F$ a multiset $\lambda(v)$ of weight symbols from $\mathbf{w}$. With each labelled shape $(F(\mathbf{x}), \lambda)$ we associate a basic expression, defined as follows. For each node $v$ of $F$ arbitrarily choose a leaf $x$ of $F(\mathbf{x})$ and number $i \geqslant 0$ such that $v=$ parent $^{i}(x)$. Let $\lambda_{v}(\mathbf{x})$ be the expression $\prod_{w \in \lambda(v)} w\left(\right.$ parent $\left.^{i}(x)\right)$. Define the $\Sigma(\mathbf{w})$-expression $F^{\lambda}(\mathbf{x})$ as the product of the expression $[F(\mathbf{x})]$ and of the all expressions $\lambda_{v}(\mathbf{x})$, for $v$ ranging over the nodes of $F$. We call $F^{\lambda}(\mathbf{x})$ the basic expression associated to $(F(\mathbf{x}), \lambda)$.

The following lemma is an immediate consequence of Lemma 30. 
Lemma 31. Let $(F(\mathbf{x}), \lambda)$ be a labelled shape and $f=\sum_{\mathbf{x}} F^{\lambda}(\mathbf{x})$. For every forest $\mathcal{A}$ and tuple of weight functions $\mathbf{w}$,

$$
f_{\mathcal{A}(\mathbf{w})}=\sum_{\alpha: F \rightarrow \mathcal{A}} \prod_{v \in F} \lambda_{v}(\alpha(v))
$$

where the sum ranges over all embeddings of rooted forests.

An $\mathbf{S}$-combination of expressions $\alpha_{1}, \ldots, \alpha_{k}$ is an expression of the form $s_{1} \cdot \alpha_{1}+\ldots+$ $s_{k} \cdot \alpha_{k}$, for $s_{1}, \ldots, s_{k} \in \mathbf{S}$. We say that two expressions $f, g$ are equivalent over a class $\mathscr{C}$ if $f_{\mathcal{A}(\mathbf{w})}=g_{\mathcal{A}(\mathbf{w})}$ for every $\mathcal{A} \in \mathscr{C}$. We also say in this case that the identity $f=g$ holds (over $\mathscr{C})$.

Lemma 32. Every sum-free $\Sigma(\mathbf{w})$-expression $\alpha(\mathbf{x})$ is equivalent over $\mathscr{C}$ to an $\mathbf{S}$-combination of basic expressions.

Proof. Fix x. The following identities hold over $\mathscr{C}$ :

$$
\begin{gathered}
{\left[\operatorname{parent}^{i}(x)=\operatorname{parent}^{j}(y)\right]+\left[\operatorname{parent}^{i}(x) \neq \operatorname{parent}^{j}(y)\right]=1} \\
{[\operatorname{root}(x)]+[\operatorname{root}(\operatorname{parent}(x))]+\left[\operatorname{root}\left(\operatorname{parent}^{2}(x)\right)\right]+\cdots+\left[\operatorname{root}\left(\operatorname{parent}^{d}(x)\right)\right]=1,} \\
{\left[\tau_{1}\left(\operatorname{parent}^{i}(x)\right)\right]+\left[\tau_{2}\left(\operatorname{parent}^{i}(x)\right)\right]+\ldots+\left[\tau_{k}\left(\operatorname{parent}^{i}(x)\right)\right]=1}
\end{gathered}
$$

where $0 \leqslant i, j \leqslant d$ and $x, y \in \mathbf{x}$, and $\tau_{1}(x), \ldots, \tau_{k}(x)$ are all formulas of the form

$$
\bigwedge_{U \in \Sigma_{+}} U(x) \wedge \bigwedge_{U \in \Sigma_{-}} \neg U(x)
$$

where $\Sigma_{+}$and $\Sigma_{-}$is a partition of the unary predicates in $\Sigma$.

Let $\gamma(\mathbf{x})$ be the product of all the left-hand sides of the above identities. Hence, $\gamma(\mathbf{x})=1$ is an identity that holds over $\mathscr{C}$, and so is $\alpha(\mathbf{x}) \cdot \gamma(\mathbf{x})=\alpha(\mathbf{x})$. Using distributivity, rewrite $\alpha(\mathbf{x}) \cdot \gamma(\mathbf{x})$ as a sum of products. Then, each summand $P(\mathbf{x})$ is a product of weight symbols applied to terms, and of expressions of the form $[\alpha]$, such that for each of the equations (8),(9),(10), exactly one of the summands on the left-hand side is present in the product $P(\mathbf{x})$. It is not difficult to see that either $P(\mathbf{x})$ is unsatisfiable in $\mathscr{C}$, in the sense that $P_{\mathcal{A}(\mathbf{w})}=0$ for every $\mathcal{A} \in \mathscr{C}$, or is of the form $F^{\lambda}(\mathbf{x})$, for some labelled shape $(F(\mathbf{x}), \lambda)$. This yields the statement.

Proof (of Lemma 29). Without loss of generality we may assume that $f$ is a sum of expressions of the form $\sum_{\mathbf{x}} \psi(\mathbf{x})$, where $\psi(\mathbf{x})$ is sum-free. By Lemma 32 we may further assume that $\psi(\mathbf{x})$ as an $\mathbf{S}$-combination of basic expressions. It is therefore enough to 
prove the lemma under the assumption that $\psi(\mathbf{x})$ is a basic expression, since we can easily construct a circuit for an S-combination of expressions, given circuits for each of them.

Hence, it suffices to prove the lemma in the case when $f$ is of the form $\sum_{\mathbf{x}} F^{\lambda}(\mathbf{x})$, for some labelled shape $(F(\mathbf{x}), \lambda)$ and variables $\mathbf{x}=\left\{x_{1}, \ldots, x_{k}\right\}$. Without loss of generality we may assume that in the shape $F(\mathbf{x})$, every node $x \in \mathbf{x}$ is distinct; otherwise we can decrease $|\mathbf{x}|$.

The proof proceeds by induction on $d$. In the base case, assume that $d=0$. If $F$ has depth larger than 0 then $f=0$ over $\mathscr{C}$. since there is no embedding $F \rightarrow \mathcal{A}$. Otherwise, $F(\mathbf{x})$ consists only of the roots $x_{1}, \ldots, x_{k}$. As for $\mathcal{A} \in \mathscr{C}$, embeddings $\alpha: F \rightarrow \mathcal{A}$ correspond to sequences $a_{1}, \ldots, a_{k}$ of distinct elements of $\mathcal{A}$, by Lemma 31 the following identity holds over $\mathscr{C}$ :

$$
f=\sum_{\substack{v_{1}, \ldots, v_{k} \\ \text { distinct }}} \lambda_{x_{1}}\left(v_{1}\right) \cdots \lambda_{x_{k}}\left(v_{k}\right) .
$$

This is the permanent of the matrix with $k$ rows and columns indexed by the vertices of $\mathcal{A}$, where the entry $(i, v)$ has value $\lambda_{x_{i}}(v)=\prod_{w \in \lambda\left(x_{i}\right)} f(v)$. This naturally yields a circuit $C_{\mathcal{A}}$, with one permanent gate connected whose inputs are outputs of bounded size circuits computing the products $\lambda_{x_{i}}(v)$, for each $v \in V$ and $1 \leqslant i \leqslant k$. Clearly, $C_{\mathcal{A}}$ can be computed in linear time, given $\mathcal{A} \in \mathscr{C}$.

In the inductive step, assume that $\mathscr{C}$ is the class of forests of depth at most $d+1$. For a forest $\mathcal{A} \in \mathscr{C}$ and its root $v$, by $\mathcal{A}^{v}$ we denote the subforest of $\mathcal{A}$ induced by the strict descendants of $v$ in $\mathcal{A}$. Similarly, for a root $r \in F$ of a shape $F(\mathbf{x})$, let $F^{r}$ denote the shape formed by the forest induced by the strict descendants of $r$ in $F$, and let $\mathbf{x}_{r}$ denote the set of nodes in $F[\mathbf{x}]$ which belong to $F^{r}$, and let $\lambda^{r}$ denote the labelling $\lambda$ restricted to $F^{r}$. Let

$$
f^{r}=\sum_{\mathbf{x}_{r}}\left(F^{r}\right)^{\lambda^{r}}\left(\mathbf{x}_{r}\right)
$$

Claim 1. The following holds for all $\mathcal{A} \in \mathscr{C}$ :

$$
f=\sum_{\beta} \prod_{r} \lambda_{r}(\beta(r)) \cdot f_{\mathcal{A}^{\beta(r)}}^{r}
$$

where $\beta$ ranges over all injective mappings of the roots of $F$ to the roots of $\mathcal{A}$ and $r$ ranges over all roots of $F$.

Proof. We use Lemma 31 to express the left-hand side above. Every embedding $\alpha: F \rightarrow$ $\mathcal{A}$ can be constructed in two steps: first choose an injection $\beta$ of the roots of $F$ into the roots of $\mathcal{A}$, and then, for each root $r$ of $F$, choose an embedding of $F^{r}$ into $\mathcal{A}^{\alpha(r)}$. Hence, 
by Lemma 31 we get:

$$
\begin{aligned}
f_{\mathcal{A}(\mathbf{w})} & =\sum_{\alpha: F \rightarrow \mathcal{A}} \prod_{v \in F} \lambda_{v}(\alpha(v)) \\
& =\sum_{\beta} \prod_{r}\left(\sum_{\alpha_{r}: F^{r} \rightarrow \mathcal{A}^{\beta(r)}} \prod_{v \in F} \lambda_{v}(\alpha(v))\right) \\
& =\sum_{\beta} \prod_{r} \lambda_{r}(\beta(r))\left(\sum_{\alpha_{r}: F^{r} \rightarrow \mathcal{A}^{\beta(r)}} \prod_{v \in F^{r}} \lambda_{v}(\alpha(v))\right)= \\
& =\sum_{\beta} \prod_{r} \lambda_{r}(\beta(r)) \cdot f_{\mathcal{A}^{\beta(r)}}^{r} .
\end{aligned}
$$

This yields the claim.

To obtain the desired circuit $C_{\mathcal{A}}$ computing $f$ on $\mathcal{A}$, we apply the inductive assumption to each expression $f^{r}$, yielding a linear time algorithm for each root $r$ of $F$, which for each forest $H$ of depth $d$ produces a circuit $C_{H}^{r}$ computing $f^{r}$. Given a forest $\mathcal{A}$ of depth $d+1$, we construct the circuit $C_{\mathcal{A}}$ which (as a term) is a permanent with rows corresponding to the roots $r$ of $F$ and columns corresponding to the roots $v$ of $\mathcal{A}$, and where the entry at row $r$ and column $v$ is the product of $\lambda_{r}(v)$ and $C_{\mathcal{A}^{v}}^{r}$. It is easy to see that that this can be implemented in linear time. Correctness of the construction follows from Claim 1.

\section{A.3 Binary relational structures of bounded treedepth}

In this section, we prove a special case of Theorem 6 , in the case when $\mathscr{C}$ is a class of structures over a binary relational signature.

Lemma 33. Let $\mathscr{C}$ be a class binary relational structures of bounded treedepth. Then $\mathscr{C}$ reduces to a class of labelled rooted forests of bounded depth.

Proof. Given a structure $\mathcal{A} \in \mathscr{C}$, let $F_{\mathcal{A}}$ be an arbitrarily chosen rooted spanning forest of the Gaifman graph of $\mathcal{A}$. As those Gaifman graphs have bounded treedepth, they also have bounded path length, so $\left\{F_{\mathcal{A}}: \mathcal{A} \in \mathscr{C}\right\}$ is a class of forests of depth bounded by some constant $d \in \mathbb{N}$.

Moreover, label every node $v$ of $F_{\mathcal{A}}$ by the following predicates, where $\Sigma$ denotes the signature of $\mathscr{C}$ :

- a unary predicate $U_{i}$, where $0 \leqslant i \leqslant d$ is the depth of $v$ in $F_{\mathcal{A}}$,

- each unary predicate $U \in \Sigma$ such that $U_{\mathcal{A}}(v)$ holds,

- for each binary symbol $R$ and $0 \leqslant i \leqslant d$, label $a$ by a new unary predicate $R_{i}$ if $R_{\mathcal{A}}(a, b)$ holds, where $b$ is the ancestor of $a$ at depth $i$ in $F_{\mathcal{A}}$. 
Finally, let $F_{\mathcal{A}}$ be equipped with the function mapping each non-root node to its parent, and the roots to themselves. This turns $F_{\mathcal{A}}$ into a labelled forest. Let $\left.\mathscr{F}=\left\{F_{\mathcal{A}}\right): \mathcal{A} \in \mathscr{C}\right\}$ be the class of all such forests.

Note that the following equivalences hold:

$$
\begin{aligned}
R(a, b) & \Longleftrightarrow \bigvee_{0 \leqslant i \leqslant j \leqslant d} U_{i}(a) \wedge U_{j}(b) \wedge\left(a=\text { parent }^{j-i}(b)\right) \wedge R_{i}(b) \\
& \vee \bigvee_{0 \leqslant i \leqslant j \leqslant d} U_{i}(b) \wedge U_{j}(a) \wedge\left(b=\text { parent }^{j-i}(a)\right) \wedge R_{i}(a) .
\end{aligned}
$$

Hence, the function mapping $\mathcal{A}$ to $F_{\mathcal{A}}$ yields a reduction from $\mathscr{C}$ to $\mathscr{F}$.

By Lemma 27, we get:

Corollary 34. The statement of Theorem 6 holds when $\mathscr{C}$ is a class of binary relational structures of bounded treedepth and $f$ is quantifier-free and all weight functions have arity 1.

\section{A.4 Classes of binary relational structures of bounded expansion}

Lemma 35. The statement of Theorem 6 holds when $\mathscr{C}$ is a class of binary relational structures of bounded expansion and $f$ is quantifier-free.

Proof. Let $f$ be a closed quantifier-free $\Sigma(\mathbf{w})$-expression. We show that there is a linear time algorithm producing circuits that evaluate $f$ over $\mathscr{C}$. It is enough to consider the case when $f$ is of the form $\sum_{\mathbf{x}} g(\mathbf{x})$ for some sum-free $\Sigma(\mathbf{w})$-expression $g$ with free variables $\mathbf{x}$. Let $p=|\mathbf{x}|$.

As $\mathscr{C}$ has bounded expansion, there is a number $d \in \mathbb{N}$ and a finite set of colors $C$ such that every structure $\mathcal{A} \in \mathscr{C}$ has a coloring $\gamma: \mathcal{A} \rightarrow C$ with colors from $C$ such that for any set $D \subseteq C$ of at most $p$ colors, the subgraph $G_{D}$ of the Gaifman graph of $\mathcal{A}$ induced by the set $\gamma^{-1}(D)$ of vertices of color in $D$ has treedepth bounded by $d$. Moreover, we can assume that the coloring $c$ as above can be computed in linear time, given $\mathcal{A} \in \mathscr{C}$.

Given a $\Sigma$-structure $\mathcal{A}$, first define its extension $\mathcal{A}^{\prime}$ by adding unary predicates $U_{c}$, for each $c \in C$, where $U_{c}$ marks the vertices of color $c$, i.e. $\gamma^{-1}(\{c\})$. For an $\mathbf{x}$-tuple of colors $\mathbf{c} \in C^{\mathbf{x}}$, define the formula testing that each variable $x$ gets color $\mathbf{c}(x)$ under $f$ :

$$
\tau_{\mathbf{c}}(\mathbf{x})=\bigwedge_{x \in \mathbf{x}} U_{\mathbf{c}[x]}(x) .
$$

Note that for every tuple $\mathbf{a} \in \mathcal{A}^{\mathbf{x}}$ there is exactly one $\mathbf{c} \in C^{\mathbf{x}}$ such that $\tau_{\mathbf{c}}(\mathbf{a})$ holds (namely, c satisfying $\mathbf{c}[x]=\gamma(\mathbf{a}[x]))$ for $x \in \mathbf{x})$. Hence, we have the following identities:

$$
\sum_{\mathbf{x}} \psi(\mathbf{x})=\sum_{\mathbf{c} \in C^{\mathbf{x}}} \sum_{\mathbf{x}}\left[\tau_{\mathbf{c}}(\mathbf{x})\right] \cdot \psi(\mathbf{x})=\sum_{\substack{D \subseteq C \\|D| \leqslant p}} \underbrace{\sum_{\mathbf{x}} \sum_{\substack{\mathbf{c} \in D^{\mathbf{x}} \\ \text { surjective }}}\left[\tau_{\mathbf{c}}(\mathbf{x})\right] \cdot \psi(\mathbf{x})}_{f^{D}} .
$$


For a set $D \subseteq C$ of at most $p$ colors, let $\mathcal{A}^{D}$ denote the substructure of $\mathcal{A}^{\prime}$ induced by $G_{D}$, and let $f^{D}$ be the expression marked above. From the above we have the following identity over $\mathscr{C}$ :

$$
f=\sum_{\substack{D \subseteq C \\|D| \leqslant p}} f^{D} .
$$

Let $\Sigma^{\prime}$ be the signature extending $\Sigma$ by the unary predicates $U_{c}$, for $c \in C$. Let $\mathscr{D}$ be the class of $\Sigma^{\prime}$-structures whose Gaifman graph has treedepth bounded by $d$. Applying Corollary 34 to $\mathscr{D}$ and $f^{D}$, for each $D \subseteq C$ of size at most $p$, we get a linear time algorithm computing circuits that evaluate $f^{D}$ on $\mathscr{D}$.

Given $\mathcal{A} \in \mathscr{C}$, we construct a circuit $C_{\mathcal{A}}$ computing $f_{\mathcal{A}}$ as follows. First compute the coloring $c: \mathcal{A} \rightarrow C$, producing a $\Sigma^{\prime}$-structure $\mathcal{A}^{\prime}$ extending $\mathcal{A}$ by the unary predicates $U_{c}$ marking the colors. Next, for each $D \subseteq C$ of size at most $p$, apply the linear time algorithm to the substructure of $\mathcal{A}^{\prime}$ induced by $c^{-1}(D)$, yielding a circuit $C_{\mathcal{A}}^{D}$ computing $f^{D}$. The resulting circuit $C_{\mathcal{A}}$ is the sum of the the circuits $C_{\mathcal{A}}^{D}$. Correctness of the construction follows from (13).

Corollary 36. Theorem 6 holds when $\mathscr{C}$ is a class of binary relational structures of bounded expansion and all weight functions have arity 1.

\section{A.5 General case}

We now treat the general case of a class of structures $\mathscr{C}$ of bounded expansion, by reducing it to the case treated previously.

We first recall the notion of degeneracy. A graph $G$ is $d$-degenerate if its edges can be oriented yielding an acyclic orientation of out-degree bounded by $d$. A class of graphs $\mathscr{G}$ has bounded degeneracy if there is some $d \in \mathbb{N}$ such that every $G \in \mathscr{G}$ is $d$-degenerate. It is well-known that a class of graphs $\mathscr{G}$ has bounded degeneracy if and only if there is a bound $c$ such that for every subgraph $H$ of a graph $G \in \mathscr{G}, H$ has at most $c \cdot|H|$ edges (cf. eg. [7]). In particular, if $\mathscr{G}$ has bounded expansion, then $\mathscr{G}$ has bounded degeneracy.

The following lemma allows us to reduce statements concerning arbitrary signatures to analogous statements concerning signatures with unary relation and function symbols only.

Lemma 37. Let $\mathscr{C}$ be a class of $\Sigma$-structures of bounded expansion, where $\Sigma$ is a relational signature. Let $\mathbf{w}$ be a set of weight symbols. There is a signature $\Sigma^{\prime}$ consisting of unary relation and function symbols, a set $\mathbf{w}^{\prime}$ of unary weight symbols, a collection of positive quantifier-free $\Sigma^{\prime}$-formulas $\left(\alpha^{R}\right)_{R \in \Sigma}$ and $\Sigma^{\prime}\left(\mathbf{w}^{\prime}\right)$-weighted expressions $\left(f^{w}\right)_{w \in \mathbf{w}}$, and an algorithm which inputs a $\Sigma(\mathbf{w})$-structure $\mathcal{A}(\mathbf{w})$ with $\mathcal{A} \in \mathscr{C}$ and computes in linear time a 
$\Sigma^{\prime}\left(\mathbf{w}^{\prime}\right)$-structure $\mathcal{A}^{\prime}$ with the same Gaifman graph as $\mathcal{A}$, such that

$$
\begin{array}{ll}
R_{\mathcal{A}}=\alpha_{\mathcal{A}^{\prime}}^{R} & \text { for each } R \in \Sigma, \\
w_{\mathcal{A}}=f_{\mathcal{A}^{\prime}\left(\mathbf{w}^{\prime}\right)}^{w} & \text { for each } w \in \mathbf{w} .
\end{array}
$$

Proof (of Lemma 37). Since $\mathscr{C}$ has bounded expansion, the class of its Gaifman graphs is $d$-degenerate, for some $d \in \mathbb{N}$. The signature $\Sigma^{\prime}$ contains unary function symbols $f_{1}, \ldots, f_{d}$, as well as unary predicates $R_{\mathbf{t}}$, for every relation symbol $R \in \Sigma$ of arity $k$ and tuple $\mathbf{t} \in[d]^{k}$.

Given a structure $\mathcal{A} \in \mathscr{C}$, fix an acyclic orientation of the edges of the Gaifman graph of $\mathcal{A}$ with out-degree at most $d$. It is well-known that such an orientation can be computed in linear time, by a greedy algorithm.

For $a \in \mathcal{A}$ and $1 \leqslant i \leqslant d+1$, let $f_{i}(a)$ be the $i$ th out-neighbor of $a$, if it exists, and $a$ otherwise. For $R \in \Sigma$ of arity $k$ and $\mathbf{t} \in[d+1]^{k}$, where $[d+1]=\{1, \ldots, d+1\}$, let $R_{\mathbf{t}}(a)$ hold if $R\left(f_{\mathbf{t}(1)}(a), \ldots, f_{\mathbf{t}(k)}(a)\right)$ holds.

Define $\mathcal{A}^{\prime}$ as the resulting $\Sigma^{\prime}$-structure consisting of the domain of $\mathcal{A}$, the unary functions $f_{1}, \ldots, f_{d}$ and unary relations $R_{\mathbf{t}}$ defined above. Then $\mathcal{A}^{\prime}$ has the same Gaifman graph as $\mathcal{A}$.

Note that for every relation $R \in \Sigma$ and tuple $\mathbf{a} \in R_{\mathcal{A}}$, all vertices in a are contained in a clique, and hence, as the chosen orientation is acyclic, there is a (unique) vertex $a$ in a such that all the vertices in $\mathbf{a}$ are of the form $f_{i}(a)$, for some (unique) $1 \leqslant i \leqslant d+1$. Hence,

$$
R\left(a_{1}, \ldots, a_{k}\right) \Longleftrightarrow \bigvee_{1 \leqslant i \leqslant k} \bigvee_{\mathbf{t} \in[d+1]^{k}} R_{\mathbf{t}}\left(a_{i}\right) \wedge \bigwedge_{1 \leqslant j \leqslant k} a_{j}=f_{\mathbf{t}(j)}\left(a_{i}\right)
$$

Similarly, for $w \in \mathbf{w}$ of arity $k$ and $\mathbf{t} \in[d+1]^{k}$, define a weight function by

$$
w_{\mathbf{t}}(a)=w\left(f_{\mathbf{t}(1)}(a), \ldots, f_{\mathbf{t}(k)}(a)\right) .
$$

Then the following equality holds:

$$
w\left(a_{1}, \ldots, a_{k}\right)=\sum_{1 \leqslant i \leqslant k} \sum_{\mathbf{t} \in[d+1]^{k}} w_{\mathbf{t}}\left(a_{i}\right) \cdot \prod_{1 \leqslant j \leqslant k}\left[a_{j}=f_{\mathbf{t}(j)}\left(a_{i}\right)\right] .
$$

This finishes the proof of Lemma 37.

Theorem 6 now follows:

Proof (of Theorem 6). We assume that $\Sigma$ contains no function symbols, as those can be encoded by relation symbols in the usual way. Hence, $\mathscr{C}$ is a class of relational structures which has bounded expansion. Applying Lemma 37 we may in turn assume that $\Sigma$ consists of unary relation and function symbols, and that all the weight symbols in $\mathbf{w}$ have arity 1 . Using the same approach as above, this can be further reduced to a class of binary relational structures, by replacing functions by their graphs. The theorem follows by Corollary 36 . 


\section{B Remaining proofs}

In this Appendix, we provide the missing details for Sections 4-7.

\section{B.1 Proof of Lemma 15}

Proof. For a fixed number of rows $k$, we use the following inclusion-exclusion formula:

$$
\sum_{\substack{x_{1}, \ldots, x_{k} \\ \text { distinct }}} w_{1}\left(x_{1}\right) \cdots w_{k}\left(x_{k}\right)=\sum_{x_{1}, \ldots, x_{k}} w_{1}\left(x_{1}\right) \cdots w_{k}\left(x_{k}\right) \prod_{i \neq j}\left(1-\left[x_{i}=x_{j}\right]\right) .
$$

Using distributivity, and then eliminating each variable $y$ occurring in an expression $[x=y]$, allows to express the permanent as a term $t$ depending on $k$ only, applied to expressions with one variable, of the form

$$
\sum_{x} w_{i_{1}}(x) \cdots w_{i_{l}}(x)
$$

where $1 \leqslant i_{1}<\ldots<i_{l} \leqslant k$. When $x$ in the sum ranges over a fixed set of $n$ elements, the above term can be viewed as a circuit $C_{n}$ which has the properties required by the lemma.

\section{B.2 Proof of Lemma 18}

Fix a finite semiring $\mathbf{S}$. By a boolean we mean either of the elements $0,1 \in \mathbf{S}$. A test gate is a gate parameterized by an element $s \in \mathbf{S}$ which inputs an element $t$ of $\mathbf{S}$ and outputs the boolean $1 \in \mathbf{S}$ if $s=t$ and $0 \in \mathbf{S}$ otherwise. A $\bmod$ gate with modulus $m \in \mathbb{N}$, inputs boolean values and outputs 1 if the number of 1 's on input is a multiple of $m$, and outputs 0 otherwise. A threshold gate has an associated threshold $t \in \mathbb{N}$, and given $n$ booleans on input, outputs 1 if at least $t$ of them are 1's. A circuit with counting gates is a circuit without permanent gates, but with test gates, mod gates and threshold gates, and well as addition and product gates.

For an integer $n$ and element $s \in \mathbf{S}$, let $n \cdot s$ denote the $n$-fold sum $s+\cdots+s$ if $n \geqslant 1$, and 0 otherwise. We will use the following lemma, providing circuits computing $\left(\sum_{i} a_{i}-k\right) \cdot s$, where $a_{1}, \ldots, a_{n}$ are input booleans and $k \geqslant 0$ and $s \in \mathbf{S}$ are fixed. Let $m_{\mathbf{S}}$ be the least common multiple of the orders of the cyclic groups contained in $(\mathbf{S},+)$. For example, for the boolean semiring, $m_{\mathbf{B}}$ is 1 and for the ring $\mathbf{Z}_{k}$ of integers modulo $k, m_{\mathbf{Z}_{k}}$ is $k$.

Lemma 38. For each fixed $s \in \mathbf{S}$ and $k \in \mathbb{N}$ there is a family $\left(C_{n}\right)_{n \in \mathbb{N}}$ of circuits with counting gates, where $C_{n}$ has $n$ boolean inputs and outputs $(l-k) \cdot s$, where $l$ is the number of 1 's among the inputs. The circuits $\left(C_{n}\right)_{n \in \mathbb{N}}$ have bounded depth, bounded fan-out, bounded expansion, each threshold is at most $\mathcal{O}(|\mathbf{S}|+k)$, and the modulus of each mod gate is the order of some cyclic subgroup of $(\mathbf{S},+)$. 
Proof (Sketch). The idea is that by finiteness of $\mathbf{S}$, the sequence $(m \cdot s)_{m \geqslant 0}$ is ultimately periodic, where the period forms a cyclic subgroup of $(\mathbf{S},+)$.

More precisely, define a directed graph $G=(V, E)$ with $V=\{0, s, 2 \cdot s, \ldots\} \subseteq \mathbf{S}$ and $E=\{(n \cdot s,(n+1) \cdot s): n \geqslant 0\}$. Since every vertex is reachable from $0 \in V$ and every vertex has one out-neighbor, the graph $G$ is a "lasso" of the form: a directed path $P$ of length at most $|\mathbf{S}|-1$, a directed cycle $C$ disjoint from $P$, and an edge connecting the endpoint of $P$ with a vertex in $C$.

Claim 2. The cycle $C$ forms a cyclic subgroup of $\mathbf{S}$.

Proof. The set $\{s, 2 \cdot s, 3 \cdot s, \ldots\}$ forms a finite sub-semigroup of $\mathbf{S}$. It is well-known that every finite semigroup contains an idempotent $e$, i.e., an element such that $e=e+e$ (specifically, $|\mathbf{S}|$ ! $\cdot s$ is such an idempotent). Let $\omega>0$ be such that $e=\omega \cdot s$ is idempotent. Then $e$ belongs to the cycle $C$, since $e=\omega \cdot s=(2 \omega) \cdot s=(3 \omega) \cdot s=\ldots$

Then $C$ forms a subgroup of $(\mathbf{S},+)$, with neutral element $e$ and inverse of $(\omega+l) \cdot s$ being $(c \cdot \omega-l) \cdot s$, where $c \in \mathbb{N}$ is such that $l \leqslant(c-1) \cdot \omega$. Moreover, $C$ is a cyclic group, generated by $(\omega+1) \cdot s$.

Let $n_{0}=|P|$. Then the elements $\left\{n \cdot s: 0 \leqslant n<n_{0}\right\}$ are all the distinct elements of the path $P$, and the elements $\left\{n \cdot s: n_{0} \leqslant n<n_{0}+|C|\right\}$ are all the distinct elements of the cycle $C$.

Fix an element $t \in \mathbf{S}$. Let $X_{t}=\{l \geqslant 0: l \cdot s=t\}$. It is easy to see that:

- if $t \notin P \cup C$ then $X_{t}=\emptyset$,

- if $t \in P$ then $X_{t}=\{n\}$, where $0 \leqslant n<n_{0}$ is such that $n \cdot s=t$,

- if $t \in C$ then $\left\{l \geqslant n_{0}:\left(l-n_{0}\right) \bmod |C|=p\right\}$, where $0 \leqslant p<|C|$ is such that $t=(l+p) \cdot s$.

For fixed $k \in \mathbb{N}$, denote by $X_{t}+k \subseteq \mathbb{N}$ the set $\left\{l+k: l \in X_{t}\right\}$. Then $X_{t}+k=$ $\{l:(l-k) \cdot s=t\}$. For each of the three cases considered in the items above, we can easily construct a family of $\left(C_{n}^{t}\right)_{n \geqslant 0}$ of circuits with threshold gates and mod gates, where $C_{n}^{t}$ inputs $n$ bits and outputs 1 if the number of 1 's among its inputs belongs to $X_{t}+k$. These circuits use mod gates with modulus $|C|$ and threshold gates with threshold $\mathcal{O}(|\mathbf{S}|+k)$.

Having constructed such circuit families $C_{n}^{t}$ for each $t \in \mathbf{S}$, we construct a circuit $C_{n}$ whose output gate is the sum of the products $t \cdot C_{n}^{t}$, for $t \in \mathbf{S}$. The circuit family $C_{n}$ has the desired properties.

Proof (of Lemma 18). Observe that the permanent of a $k \times n$ matrix $M$ can be computed based on the number of occurrences of each tuple $c \in \mathbf{S}^{k}$ as a column in $M$, as follows. Fix a matrix $k \times k$ matrix $N$. For each $i \in[k]$, let $d_{i}$ be the $i$ th diagonal entry of $N$, let $c_{i}$ be the $i$ th column of $N$, let $n_{i}$ be the number of occurrences of $c_{i}$ in $N$ and let $m_{i}$ be the 
number of occurrences of $c_{i}$ in $M$. Finally, let $s_{i}$ be the product $m_{i} \cdots\left(m_{i}-n_{i}+1\right) \cdot d_{i} \in \mathbf{S}$, and let $p_{N}$ be the product $s_{1} \cdots s_{k}$. The permanent of $M$ is equal to the sum of all the values $p_{N}$, for $N$ ranging over all $k \times k$ matrices. Note that the value $s_{i}$ can be computed by applying $n_{i}$ times Lemma 38 . This yields the required circuit family.

\section{B.3 Proof of Theorem 22}

Proof (of Lemma 23). Let $M$ be an $R \times C$ matrix and let $r \in R$ be an arbitrarily chosen row. Observe that the following identity holds:

$$
\operatorname{perm}(M)=\sum_{c \in C} M[r, c] \cdot \operatorname{perm}\left(M^{r c}\right),
$$

where $M^{r c}$ is the matrix $M$ with row $r$ and column $c$ removed. The idea is to recursively use the above identity to enumerate $\operatorname{perm}(M)$. For this to work, we need to iterate over all columns $c$ such that the product $M[r, c] \cdot \operatorname{perm}\left(M^{r c}\right)$ is nonempty.

To this end, observe that the function $h: \mathbf{F}_{A} \rightarrow \mathbf{B}$ which maps 0 to 0 and every non-zero element in $\mathbf{F}_{A}$ to 1, is a semiring homomorphism. It follows that $h(\operatorname{perm}(A))=\operatorname{perm}(h(A))$, for any matrix $A$ with entries in $\mathbf{F}_{A}$ and matrix $h(A)$ obtained from $A$ by mapping each entry via $h$.

Let $N$ denote the matrix $h(M)$, and let $N^{r c}$ denote the matrix obtained by removing the row $r$ and column $c$. Thanks to the above, the problem mentioned earlier boils down to the problem considered in the following lemma.

Lemma 39. There is a dynamic algorithm which inputs an $R \times C$ boolean matrix $N$ and a row $r$, and computes in linear time a data structure which maintains in constant time a bi-directional iterator for the set of those columns $c \in C$ with $N[r, c]=\operatorname{perm}\left(N^{r c}\right)=1$.

Finally, to enumerate perm $(M)$, we use (14). Fix $r \in R$ and iterate over all columns $c$ such that $N[r, c]=\operatorname{perm}\left(N^{r c}\right)=1$. For each considered column $c$, the iterator for $M[r, c]$ is given by assumption, and the iterator for perm $\left(M^{r c}\right)$ is obtained recursively (note that $M^{r c}$ has one row fewer than $\left.M\right)$. Using them, iterate over the product $M[r, c] \cdot \operatorname{perm}\left(M^{r c}\right)$ in a lexicographic fashion.

Proof ( of Lemma 39). Our data structures stores, for each vector $\mathbf{t} \in \mathbf{B}^{S}$, a bi-directional list $L_{\mathbf{t}}$ over all occurrences of $\mathbf{t}$ as a column in $N$.

Note that this data structure can computed in time $\mathcal{O}_{R}(|C|)$. Moreover, it can be maintained in time $\mathcal{O}_{R}(1)$, upon an update to $N$ : if an update affects an entry at row $r$ and column $c$, then we remove this column from the lists it belonged to, and append it to the appropriate list.

Let $K \subseteq \mathbf{B}^{S}$ be the set of those vectors $\mathbf{t}$ which occur in $N$ as some column $c$ such that $N[r, c]=\operatorname{perm}\left(N^{r c}\right)=1$. Note that this property does not depend on the choice of the column $c$ among all occurrences of $\mathbf{t}$. 
Moreover, $K$ can be computed only basing on the number of occurrences of each vector $\mathbf{t} \in \mathbf{B}^{S}$ as a column in $N$, where the number of occurrences is only counted up to $|R|$. It follows that the set $L$ can be computed in constant time, basing on the existing data structure, since we can iterate through $L_{\mathbf{t}}$ for at most $|R|$ steps to determine the number of occurrences.

This yields the desired bi-directional iterator for the columns $c$ such that $N[r, c]=$ $\operatorname{perm}\left(N^{r c}\right)=1$ : it is the concatenation of the lists $L_{\mathbf{t}}$, for $\mathbf{t} \in K$.

Proof (of Theorem 22). We give the proof in the case when $f$ is a closed expression. The general case follows as in the proof of Theorem 8 , by simulating a query by a sequence of $|\mathbf{x}|$ temporary updates.

Consider the circuit family $\left(C_{\mathcal{A}}\right)_{\mathcal{A} \in \mathscr{C}}$ given by Theorem 6 . For simplicity, and without loss of generality, assume that all inner gates in $C_{\mathcal{A}}$ are permanent gates, as multiplication and addition can be simulated by such.

To prove Theorem 22, apply Lemma 23 to each gate $g$ of $C_{\mathcal{A}}$, starting from the input gates and moving towards the output gate. This yields an iterator representing the value at the gate $g$ of $C_{\mathcal{A}}$ induced by the weight functions $\mathbf{w}$. The iterator at the output gate is the required iterator for $f_{\mathcal{A}(\mathbf{w})}$.

\section{B.4 Proof of Theorem 24}

We now consider the dynamic case of Theorem 24.

Without loss of generality, assume that the signature $\Sigma$ contains only relation symbols of arity bounded by some number $r_{\max } \in \mathbb{N}$. Also without loss of generality, we may assume that the Gaifman graph of the input structure $\mathcal{A}$ is left unchanged by all the updates. Indeed, we can assume that the input structure $\mathcal{A}$ includes a binary edge relation $E$ which initially represents exactly the Gaifman graph $G_{\mathcal{A}}$ of $\mathcal{A}$, and which is never modified by updates (the relation $E$ can be added to $\mathcal{A}$ in linear time in the preprocessing phase). Furthermore, for all $2 \leqslant r \leqslant r_{\max }$, we assume that $\mathcal{A}$ contains a relation $R_{k}$ consisting of all tuples $\mathbf{a} \in \mathcal{A}^{k}$ whose elements form a clique in $G_{\mathcal{A}}$, and that the relations $R_{1}, \ldots, R_{r}$ are never modified by updates. Henceforth, we assume that the updates never modify the relations $R_{1}, \ldots, R_{r_{\max }}$ of a structure $\mathcal{A}$. We call those updates Gaifman-preserving updates since they preserve the Gaifman graph of $\mathcal{A}$.

To employ our circuit framework, we encode the relations of $\mathcal{A}$ by weight functions with values $\{0,1\} \in \mathbf{F}$, where $\mathbf{F}$ is the free commutative semiring.

Lemma 40. Let $\Sigma, \varphi, \mathscr{C}$ be as in Theorem 24, with $\varphi(\mathbf{x})$ quantifier-free. There is a signature $\Sigma^{\prime}$, a set of weight symbols $\mathbf{v}$, a quantifier-free $\Sigma^{\prime}(\mathbf{v})$-expression $g(\mathbf{x})$, and a dynamic algorithm which, given a structure $\mathcal{A} \in \mathscr{C}$, computes in linear time a $\Sigma^{\prime}$-structure $\mathcal{A}^{\prime}$ with the same Gaifman graph as $\mathcal{A}$ and a tuple of weight functions $\mathbf{v}_{\mathcal{A}}$ with values in $\mathbf{0}, \mathbf{1}$, which interpreted in any semiring $\mathbf{S}$ satisfy

$$
g_{\mathcal{A}^{\prime}(\mathbf{v})}(\mathbf{a})=\left[\varphi_{\mathcal{A}}(\mathbf{a})\right] \quad \text { for } \mathbf{a} \in \mathcal{A}^{\mathbf{x}} .
$$


When a Gaifman-preserving update is applied to $\mathcal{A}$, then $\mathcal{A}^{\prime}(\mathbf{v})$ is updated in constant time by updating $\mathbf{v}$ and leaving $\mathcal{A}^{\prime}$ unchanged.

Proof. Given a structure $\mathcal{A}$, the structure $\mathcal{A}^{\prime}$ contains only the relations $R_{1}, \ldots, R_{r_{\max }}$ as described before the lemma. In particular, $\mathcal{A}^{\prime}$ is not modified when a Gaifman-preserving update is applied to $\mathcal{A}$.

Define a set of weight symbols $\mathbf{v}$ containing symbols $v_{R}^{+}$and $v_{R}^{-}$of arity $r$, for each relation $R \in \Sigma$ of arity $r$. For each $R \in \Sigma$ of arity $r$, define $\mathbf{S}$-weight functions $v_{R}^{+}$and $v_{R}^{-}$ of arity $r$ on $\mathcal{A}^{\prime}$ as follows:

$$
\begin{aligned}
v_{R}^{+}\left(a_{1}, \ldots, a_{k}\right) & =\left[R_{\mathcal{A}}\left(a_{1}, \ldots, a_{k}\right)\right], \\
v_{R}^{-}\left(a_{1}, \ldots, a_{k}\right) & =\left[\neg R_{\mathcal{A}}\left(a_{1}, \ldots, a_{k}\right)\right],
\end{aligned}
$$

It is clear that given $\mathcal{A}$, the structure $\mathcal{A}^{\prime}(\mathbf{v})$ can be computed in linear time, and updated in constant time, upon Gaifman-preserving updates to $\mathcal{A}$.

It remains to describe the expression $g(\mathbf{x})$ satisfying (15). Let $T$ be the set of terms occurring in $\varphi$. Rewrite $\varphi(\mathbf{x})$ as a disjunction of atomic types $\alpha(\mathbf{x})$, where an atomic type is a maximal consistent conjunction of literals involving terms from $T$ only. As any two inequivalent atomic types $\alpha(\mathbf{x})$ and $\beta(\mathbf{x})$ are mutually exclusive, it follows that $[\alpha(\mathbf{a}) \vee$ $\beta(\mathbf{a})]=[\alpha(\mathbf{a})]+[\alpha(\mathbf{a})]$, in any semiring $\mathbf{S}$. Therefore, it is enough to consider the case when $\varphi(\mathbf{x})$ is an atomic type $\varphi(\mathbf{x})=\alpha_{1}\left(t_{1}\right) \wedge \cdots \wedge \alpha_{k}\left(t_{k}\right)$, where $\alpha\left(t_{i}\right)$ are literals. Clearly, no matter what semiring $\mathbf{S}$ is considered, the following equivalence holds:

$$
\left[\alpha_{1}\left(t_{1}\right) \wedge \cdots \wedge \alpha_{k}\left(t_{k}\right)\right]=\left[\alpha_{1}\left(t_{1}\right)\right] \cdots\left[\alpha_{k}\left(t_{k}\right)\right] .
$$

Therefore, it is enough to consider the case when $\varphi(\mathbf{x})$ is a single literal $R\left(t^{1}, \ldots, t^{k}\right)$ or $\neg R\left(t^{1}, \ldots, t^{k}\right)$, for some terms $t^{1}, \ldots, t^{k} \in T$. In the first case, define $g(\mathbf{x})$ as $v_{R}^{+}\left(t^{1}, \ldots, t^{k}\right)$, and in the latter, define $g(\mathbf{x})$ as $v_{R}^{-}\left(t^{1}, \ldots, t^{k}\right)$. By construction of $\mathcal{A}^{\prime}(\mathbf{v})$, it is clear that $(15)$ holds.

Proof ( OF Theorem 24). We proceed similarly to the static case described in Section 6. We use Theorem 6.3 in [7], which extends Theorem 3 by stating that $\widehat{\mathcal{A}}$ can be updated in constant time upon a Gaifman-preserving update to $\mathcal{A}$. Thanks to this, we can assume that $\varphi(\mathbf{x})$ is quantifier-free.

Let $\mathbf{x}=\left\{x_{1}, \ldots, x_{k}\right\}$. As in the static case, define weight functions $w_{1}, \ldots, w_{k}: \mathcal{A} \rightarrow \mathbf{F}$, where $w_{i}(a)=e_{a}^{i}$. Instead of considering the expression $f$ defined in (4), we now define $f$ as follows:

$$
f=\sum_{\mathbf{x}} g(\mathbf{x}) \cdot w_{1}\left(x_{1}\right) \cdots w_{k}\left(x_{k}\right)
$$

where $g$ is given by Lemma 40. Our dynamic data structure maintains the $\Sigma^{\prime}(\mathbf{v})$-structure $\mathcal{A}^{\prime}(\mathbf{v})$ as described in the lemma. In particular, the invariant $g_{\mathcal{A}^{\prime}(\mathbf{v})}(\mathbf{x})=\left[\varphi_{\mathcal{A}}(\mathbf{x})\right]$ is maintained by updates. 
Together with the weight functions $w_{1}, \ldots, w_{k}$, this yields a $\Sigma^{\prime}(\mathbf{v w})$-structure $\mathcal{A}^{\prime}(\mathbf{v w})$. By (16), the element $f_{\mathcal{A}^{\prime}(\mathbf{v w})} \in \mathbf{F}$ is a formal sum representing the set of tuples $\varphi_{\mathcal{A}} \subseteq \mathcal{A}^{\mathbf{x}}$, and this is maintained by the updates. Applying Theorem 22 yields a bi-directional iterator with constant access time which enumerates the components of $f_{\mathcal{A}^{\prime}(\mathbf{v w})}$. More precisely, our dynamic algorithm is obtained by composing the dynamic algorithm maintaining $\mathcal{A}^{\prime}(\mathbf{v w})$ given by Lemma 40 with the dynamic data structure maintaining an enumerator for $f_{\mathcal{A}^{\prime}(\mathbf{v w})}$ given by Theorem 22. An update to $\mathcal{A}$ triggers constantly many updates to $\mathbf{v}$, and an enumerator for $f_{\mathcal{A}^{\prime}(\mathbf{v w})}$ can be maintained in constant time.

\section{B.5 Proof of Theorem 26}

Proof. We proceed by induction on the number of guarded connectives in $\varphi$ of the form $\left[R\left(x_{1}, \ldots, x_{l}\right)\right] \cdot c\left(\varphi_{1}, \ldots, \varphi_{k}\right)$. In the base case, there are no guarded connectives. If $\varphi$ is has values in $\mathbf{B}$, it is a first-order formula, and the statement follows from Theorem 3 and Theorem 24 for the enumeration part. Otherwise, $\varphi$ is an $\mathbf{S}$-valued formula, for some semiring $\mathbf{S} \in \mathbb{C}$, and $\varphi$ can be seen as a $\Sigma^{\prime}(\mathbf{w})$-expression, where $\Sigma^{\prime}$ consists of the function symbols and the $\mathbf{B}$-valued relations in $\Sigma$, and $\mathbf{w}$ consists of the $\mathbf{S}$-valued relations in $\Sigma$. In this case, the statement follows from Theorem 8.

In the inductive step, let $\varphi(\mathbf{x}) \in \mathrm{FO}_{\mathrm{G}}[\mathbb{C}]$ and suppose that the statement holds for every formula with fewer guarded connectives.

Let $\left[R\left(x_{1}, \ldots, x_{l}\right)\right] \cdot c\left(\varphi_{1}, \ldots, \varphi_{k}\right)$ be a guarded connective in $\varphi$ which does not appear in the scope of another guarded connective, and suppose $c: \mathbf{S}_{1} \times \cdots \mathbf{S}^{k} \rightarrow \mathbf{S}$. Let $\mathbf{x}=$ $\left\{x_{1}, \ldots, x_{l}\right\}$. Apply the inductive assumption to the formulas $\varphi^{1}(\mathbf{x}), \ldots, \varphi^{k}(\mathbf{x})$. Given a structure $\mathcal{A} \in \mathscr{C}$, we can compute the functions $\varphi_{\mathcal{A}}^{i}: \mathcal{A}^{\mathbf{x}} \rightarrow \mathbf{S}_{i}$ in the required time. Next, define the $\mathbf{S}$-valued relation $r: \mathcal{A}^{k} \rightarrow \mathbf{S}$ by $r(\mathbf{a})=c\left(\varphi_{\mathcal{A}}^{1}(\mathbf{a}), \ldots, \varphi_{\mathcal{A}}^{k}(\mathbf{a})\right)$ for $\mathbf{a} \in R_{\mathcal{A}} \subseteq \mathcal{A}^{\mathbf{x}}$, and $r(\mathbf{a})=0$ for $\mathbf{a} \notin R_{\mathcal{A}}$. This can be computed in linear time, by scanning through all tuples $\mathbf{a} \in R_{\mathcal{A}}$ and for each of them, applying in constant time the connective $c$ to the tuple of precomputed values.

Define the structure $\mathcal{A}^{\prime}$ as $\mathcal{A}$ extended by the $\mathbf{S}$-valued relation $r$. Replace in the formula $\varphi(\mathbf{x})$ the guarded connective $\left[R\left(x_{1}, \ldots, x_{l}\right)\right] \cdot c\left(\varphi_{1}, \ldots, \varphi_{k}\right)$ by the atom $r\left(x_{1}, \ldots, x_{l}\right)$. Clearly, $\varphi_{\mathcal{A}^{\prime}}^{\prime}=\varphi_{\mathcal{A}}$. Moreover, $\varphi^{\prime}$ has fewer guarded connectives than $\varphi$, so the conclusion follows from the inductive assumption. 